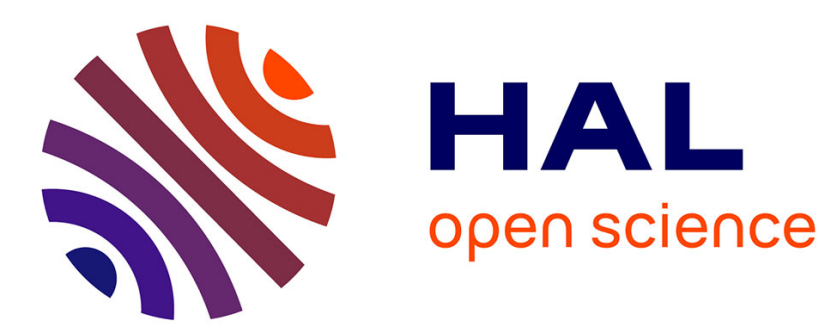

\title{
Linear Sampling Method applied to Non Destructive Testing of an elastic waveguide: theory, numerics and experiments
}

Vahan Baronian, Laurent Bourgeois, Bastien Chapuis, Arnaud Recoquillay

\section{- To cite this version:}

Vahan Baronian, Laurent Bourgeois, Bastien Chapuis, Arnaud Recoquillay. Linear Sampling Method applied to Non Destructive Testing of an elastic waveguide: theory, numerics and experiments . Inverse Problems, 2018, 10.1088/1361-6420/aac21e . hal-01816810

\author{
HAL Id: hal-01816810 \\ https://hal.inria.fr/hal-01816810
}

Submitted on 15 Jun 2018

HAL is a multi-disciplinary open access archive for the deposit and dissemination of scientific research documents, whether they are published or not. The documents may come from teaching and research institutions in France or abroad, or from public or private research centers.
L'archive ouverte pluridisciplinaire HAL, est destinée au dépôt et à la diffusion de documents scientifiques de niveau recherche, publiés ou non, émanant des établissements d'enseignement et de recherche français ou étrangers, des laboratoires publics ou privés. 


\title{
Linear Sampling Method applied to Non Destructive Testing of an elastic waveguide: theory, numerics and experiments
}

\author{
Vahan Baronian $^{\mathrm{a}}$, Laurent Bourgeois ${ }^{\mathrm{b}, *}$, Bastien Chapuis ${ }^{\mathrm{a}}$, Arnaud \\ Recoquillay ${ }^{\mathrm{a}, \mathrm{b}}$ \\ ${ }^{a}$ CEA, LIST, Gif-sur-Yvette, France \\ ${ }^{b}$ Laboratoire POEMS, UMR ENSTA/CNRS/INRIA, ENSTA ParisTech, 828 Boulevard des \\ Maréchaux, 91120 Palaiseau, France
}

\begin{abstract}
This paper presents an application of the Linear Sampling Method to ultrasonic Non Destructive Testing of an elastic waveguide. In particular, the NDT context implies that both the solicitations and the measurements are located on the surface of the waveguide and are given in the time domain. Our strategy consists in using a modal formulation of the Linear Sampling Method at multiple frequencies, such modal formulation being justified theoretically in 1 for rigid obstacles and in [2] for cracks. Our strategy requires the inversion of some emission and reception matrices which deserve some special attention due to potential ill-conditioning. The feasibility of our method is proved with the help of artificial data as well as real data.

Keywords: Elastic waveguide, Inverse scattering problem, Linear Sampling Method, Lamb modes, Experimental data.
\end{abstract}

\section{Introduction}

This paper presents a Non Destructive Testing method to identify some defects in an elastic waveguide. Such a subject is far from being original, but the novelty of our contribution is that we apply a rather mathematical approach of 5 inverse problems, namely the Linear Sampling Method, to improve the ultrasonic inspection of elastic waveguide structures from real data. In particular, at the end of the paper we present some promising identification results obtained with experimental data. There is a huge amount of works dealing with NDT experimental ultrasonic techniques for waveguides such as metallic plates or pipes. But NDT ultrasonic techniques have limitations: some of them take the modal decomposition of the waves into account, which allows the inspection

\footnotetext{
${ }^{*}$ Corresponding author

Email address: laurent.bourgeois@ensta.fr (Laurent Bourgeois)
} 
of a wide area. But these techniques need to track the propagation of a single guided mode at a low frequency, which is difficult in practice [3, 4, 5, 6, 7, 8, Moreover, the guided-wave based techniques often depend on the type of defect 15 which is expected, that is a void, a crack or some corrosion. Other techniques do not take the modal decomposition of the waves into account and enable the inspection, at a high frequency, of a limited area which is close to the sensor [9, 10. Our aim is to image an elastic waveguide in a wide area and at a high frequency, using all the propagating modes at the same time. Furthermore, we 20 wish to conceive a method which does not depend on the number and the type of defects that are likely to be detected. A sampling method such as the Linear Sampling Method has all these nice features, provided we manage to adapt it to the constraining context of NDT.

The Linear Sampling Method has now a long history since its introduction 25 in [11] in the free space configuration and for the acoustic case. It consists, for each sampling point $z$ describing a grid, to check if some appropriate and analytically known test function depending on $z$ belongs or not to the range of a linear operator the definition of which is based on the data. In the case of a positive answer, the point $z$ belongs to the defect, which is a practical way

so of retrieving its boundary. Since the nineties, the LSM has been widely used in other configurations and for other types of physical settings. For example, both for penetrable or impenetrable objects, the LSM was extended to elasticity in [12, 13] and to electromagnetism in [14, 15]. In the acoustic case, the LSM was adapted to the waveguide geometry in [16, 17, 18, 19. The case of elastic 35 waveguides was addressed in [1, the case of electromagnetic waveguides in 20. Given the importance of cracks in NDT, we mention that the LSM was adapted to the detection of cracks in 21, 22 in the case of acoustics and in 2, 23 in the case of elasticity. To complete this short review of the Linear Sampling Method, while the previous contributions consider waves in the frequency domain, we 40 point out that a time domain version of the LSM was developed in 24] and adapted to waveguides in [25].

In almost all papers cited above, the LSM was applied to artificial data which somehow can be considered as ideal. In 1, 2, 17, 25, for instance, the sources and the receivers are located along transverse sections of the waveguide, which

${ }_{45}$ is not realistic in the context of Non Destructive Testing, where the sources and the receivers are both located on the surface of the waveguide. Furthermore, 1, 2, 17, stick to the frequency regime, while data are produced in the time domain in practice. The work we propose in elasticity is the same as we did in [26] in the acoustic case starting from [18]: handling surface data in the time 50 domain in order to be in the exact conditions of a true experiment. An important contribution in [1, 2, 18, was that a modal formulation of the Linear Sampling Method, which is specific to waveguides, was introduced. Indeed, the term "modal formulation" refers to the fact that all the incident and scattered fields, in the region of the waveguide where there is no defect, can be decomposed along

55 the guided modes. This decomposition enables us to select, among all modal amplitudes of the incident and scattered fields, those which correspond to the propagating part, that is the part which propagates at long distance without 
attenuation. The remainder of the modal amplitudes is simply dropped. The advantage of such technique, which is justified in the acoustic case in [18, is that it regularizes, in a very efficient way, the ill-posed LSM equation to solve for each sampling point. In addition, our modal approach enables us to optimize the number of the sources/receivers and the spacing between them. Note that such optimization is significantly more difficult in the case of elasticity than in the case of acoustics, due to much higher complexity of the dispersion curves.

65 Like in 26], our strategy is the following. Firstly, we transform the time domain data into multi-frequency data with the help of the Fourier transform. Secondly, at each frequency we transform the surface data into a novel set of ideal data which are suitable for the modal formulation of the LSM. Thirdly, we combine all the images given by the LSM at each frequency in order to obtain the best 70 possible defect identification. The Linear Sampling Method in the frequency domain for an elastic waveguide is already justified for a Dirichlet obstacle in [1] and for cracks in [2]. The justification for a Neumann obstacle would follow the same lines and the justification for a penetrable obstacle would be very close to that of [27] for the periodic acoustic waveguide. It is important to note that our 75 article does not provide any new result in this direction. Instead, it is focused on the application of the LSM in the presence of real data (in particular, surface data in the time domain) and on how the substantial issues generated by those real data can be fixed. To our best knowledge, proving the feasibility of the LSM with the help of real experiments on an elastic waveguide is new.

80 The outline of our paper is the following. Our Non Destructive Testing problem is described in section 2. In section 3, we briefly introduce the framework of mixed $\mathbf{X}, \mathbf{Y}$ variables, which is a key tool in view of the modal formulation of the Linear Sampling Method. In section 4, we summarize such modal formulation and its far field approximation. Section 5 details the way, starting from surface

85 data in the time domain, we can handle those data in order to come back to the previous modal formulation. The section 6 is dedicated to some numerical experiments with artificial data, while section 7 presents our experimental setup and the corresponding identification results. Some conclusions are given in section 8. Lastly, in appendix A we derive the fundamental solution of the 90 elastic waveguide for a Dirac located on its boundary, while the appendix B is an analysis of the cases when the matrix of modes introduced in section 5 is not invertible.

\section{Setting of the problem}

We consider a $2 \mathrm{D}$ waveguide $W=S \times \mathbb{R}$ for $S=(0, d)$ and $d>0$ is the height

95 of the waveguide. We denote $\left(x_{1}, x_{3}\right)$ the coordinates of a generic point of $W$ in the orthonomal basis $\left(\mathbf{e}_{1}, \mathbf{e}_{3}\right), x_{3}$ being the coordinate of the infinite direction of the waveguide. The boundary of $W$ is denoted $\Gamma=\Gamma_{0} \cup \Gamma_{d}$, where $\Gamma_{0}\left(\operatorname{resp} . \Gamma_{d}\right)$ corresponds to $x_{1}=0$ (resp. $x_{1}=d$ ). This $2 \mathrm{D}$ configuration can also be viewed as a $3 \mathrm{D}$ configuration, such that if $\left(x_{1}, x_{2}, x_{3}\right)$ are the three spatial coordinates,

100 the waveguide is infinite in both directions $x_{2}$ and $x_{3}$ with invariance of all quantities with respect to $x_{2}$. This will coincide with the configuration of our 
experimental device in section 7 . In what follows, all vectors in $\mathbb{R}^{2}$ will be marked in bold characters while scalars and matrices will appear normally. For instance, $\mathbf{v}$ will denote the displacement, $\varepsilon$ and $\sigma$ the strain and stress tensors, respectively. We recall that, in the case of linear and isotropic elasticity, they are linked by

$$
\varepsilon(\mathbf{v})=\frac{\nabla \mathbf{v}+\nabla \mathbf{v}^{T}}{2}, \quad \sigma(\mathbf{v})=\lambda \operatorname{tr}[\varepsilon(\mathbf{v})] \operatorname{Id}_{2}+2 \mu \varepsilon(\mathbf{v}),
$$

where $m^{T}, \operatorname{tr}(m)$ denote the transpose and the trace of the matrix $m$, respectively, $\operatorname{Id}_{2}$ denotes the identity matrix, while $\lambda$ and $\mu$ are the Lamé constants, which satisfy $\lambda+2 \mu>0$ and $\mu>0$.

We now consider a compactly supported scalar function $\varphi$ of $x_{3}$ and a smooth (at least $C^{1}$ ) compactly supported scalar function $\chi$ of time $t$, with $\operatorname{supp}(\chi) \subset$ $[0,+\infty)$. For some impenetrable obstacle $D$ such that $\bar{D} \subset W$, let us denote $\Omega=W \backslash \bar{D}$ and assume that $\Omega$ is connected. We consider the displacement solution $\mathbf{v}$ in $\Omega \times(0,+\infty)$ to the scattering problem with Dirichlet boundary condition on $\partial D$ :

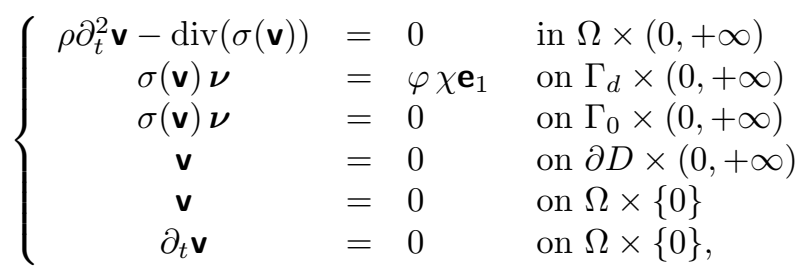

where $\rho$ is the density of the material and $\boldsymbol{\nu}$ is the outward unit normal to $\Omega$. It should be noted that $\boldsymbol{\nu}=\mathbf{e}_{1}$ on $\Gamma_{d}$ and $\boldsymbol{\nu}=-\mathbf{e}_{1}$ on $\Gamma_{0}$. Let us introduce two scalar functions $f$ and $g$ that are both compactly supported and even. While the function of time $\chi$ is given once and for all, the function of space $\varphi$ is alternatively chosen as

$$
\varphi\left(x_{3}\right)=f\left(x_{3}-x_{3}^{s \pm}\right),
$$

for several source locations $x_{3}^{s \pm}$. We denote by $\mathbf{v}^{i}$ the solution to the same problem as (1) in $W$ without the boundary condition on $\partial D$ and $\mathbf{v}^{s}=\mathbf{v}-\mathbf{v}^{i}$. In the classical terminology of scattering problems, $\mathbf{v}$ is the total field while $\mathbf{v}^{i}$ is the incident field and $\mathbf{v}^{s}=\mathbf{v}-\mathbf{v}^{i}$ is the scattered field. The measurements are given for all time $t \in(0,+\infty)$, for several receiver locations $x_{3}^{r \pm}$, by

$$
\gamma\left(x_{3}^{r \pm}, t\right)=\int_{\mathbb{R}} g\left(x_{3}-x_{3}^{r \pm}\right) \mathbf{v}_{1}^{s}\left(d, x_{3}, t\right) d x_{3},
$$

where $\mathbf{v}_{1}^{s}$ is the component of the displacement $\mathbf{v}^{s}$ along $\mathbf{e}_{1}$. We assume that the position of sources and receivers are given by

$$
x_{3}^{m \pm}= \pm(R+m \delta), \quad m=0, \ldots, M-1,
$$

where $R>0$ is such that the obstacle $D$ is a priori located between the transverse sections of coordinates $x_{3}=-R$ and $x_{3}=R$ and $\delta>0$. The well-posedness 


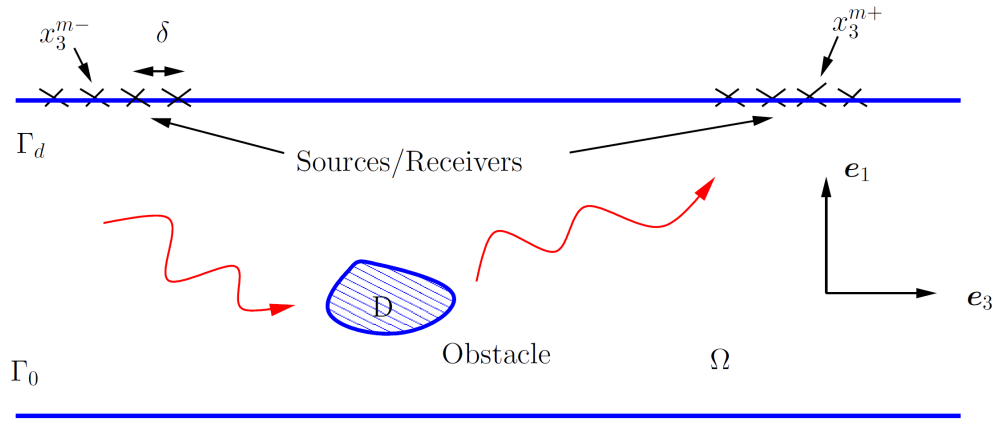

Figure 1: Configuration of the inverse problem

of the forward problem (1) for some data $(\varphi, \chi)$, which can be analyzed with classical tools like the J.-L. Lions Theorem or the Hille-Yosida Theorem (see [28], will not be discussed in the present paper. The inverse problem consists in identifying the obstacle $D$ in two situations:

- the full-scattering situation, that is we use the sources and receivers at $x_{3}^{m+}$ and $x_{3}^{m-}$ given by 4 for $m=0, \ldots, M-1$, which amounts to $2 M$ sources and $2 M$ receivers,

- the back-scattering situation, that is we use the sources at $x_{3}^{m-}$ and the receivers at $x_{3}^{m-}$ for $m=0, \ldots, M-1$, which amounts to $M$ sources and $M$ receivers.

In the full-scattering situation, both sides of the defect are accessible, while only one side is accessible in the back-scattering situation. The configuration of our inverse problem is illustrated in figure 1. In the inverse problem that we consider, the obstacle $D$ is characterized by a Dirichlet boundary condition $\mathbf{v}=0$ on $\partial D$ for simplicity, like in [1. But all the justifications remain valid for

145 the Neumann boundary condition $\sigma(\mathbf{v}) \boldsymbol{\nu}=0$ on $\partial D$ or $\sigma(\mathbf{v}) \boldsymbol{\nu}=0$ on both sides of a non closed curve, which model a void or a crack, respectively, like those we look for in the real experiment. We introduced the functions $f$ and $g$ in order to make the data of the inverse problem closer to the real data. Indeed, a Dirac Distribution would correspond for $f$ to a punctual force applied at point $x_{3}^{r \pm}$ 150 and for $g$ to a punctual measurement of the displacement at point $x_{3}^{s} \pm$, while in practice using piezoelectric transducers leads us to impose a distributed force around $x_{3}^{r \pm}$ and to measure a weighted displacement around $x_{3}^{s} \pm$. Lastly, in our inverse problem, we impose a pressure force (along $\mathbf{e}_{1}$ ) on the boundary of the waveguide and measure the transverse displacement (along $\mathbf{e}_{1}$ ) on such 155 boundary, because this is what is usually done in experiments and what we actually do in section 7 . In fact, the theory developed in the present article enables us to consider other configurations (see [29]), for example a shear force (along $\mathbf{e}_{3}$ ) on the boundary and the axial displacement (along $\mathbf{e}_{3}$ ). 


\section{The $X, Y$ variables}

In this section we briefly summarize the results given in 30 .

\subsection{The guided modes}

Let us introduce the $\mathbf{X}$ and $\mathbf{Y}$ variables, which is a useful tool in order to study the guided modes. They are defined from the displacement field $\mathbf{u}$ as

$$
\mathbf{X}=\left(\begin{array}{l}
\mathrm{t}_{1} \\
\mathrm{u}_{3}
\end{array}\right) \quad \text { and } \quad \mathbf{Y}=\left(\begin{array}{l}
\mathrm{u}_{1} \\
\mathrm{t}_{3}
\end{array}\right)
$$

where $\mathbf{u}=\left(\mathbf{u}_{1}, \mathbf{u}_{3}\right)$ and $\sigma(\mathbf{u}) \mathbf{e}_{3}=\left(\mathrm{t}_{1},-\mathrm{t}_{3}\right):=\left(\sigma_{31}, \sigma_{33}\right)$. These variables $\mathbf{X}$ and $\mathbf{Y}$, which mix some components of the displacement and some components of the stress tensor, were introduced in 31 and revisited in 30, 32. The main advantage of those variables is that, contrary to the displacement field $\mathbf{u}$, its $\mathbf{X}$ and $\mathbf{Y}$ extensions can be decomposed in a complete basis of any transverse section of the waveguide, as we will see later. In our elastic waveguide, for a given fixed frequency $\omega$, the so-called guided modes are the solutions $\mathbf{u}$, with separate dependance on $x_{1}$ and $x_{3}$, to the system

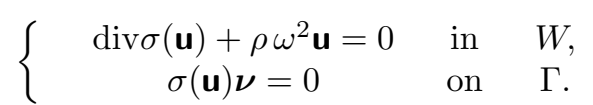

It can be proved (see [30]) by using the $\mathbf{X}, \mathbf{Y}$ variables that the system (5) is equivalent to the "evolution" problem with respect to the coordinate $x_{3}$ :

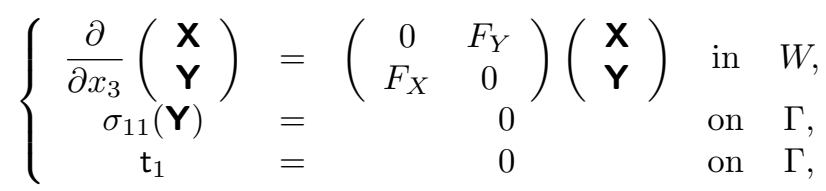

where the transverse operators $F_{X}$ and $F_{Y}$ are defined by

$$
F_{Y} \mathbf{Y}=\left(\begin{array}{c}
-\partial_{x_{1}} \sigma_{11}(\mathbf{Y})-\rho \omega^{2} \mathbf{u}_{1} \\
-\alpha \partial_{x_{1}} \mathrm{u}_{1}-\frac{\alpha}{\lambda} \mathrm{t}_{3}
\end{array}\right) \quad \text { and } \quad F_{X} \mathbf{X}=\left(\begin{array}{c}
\frac{\mathrm{t}_{1}}{\mu}-\partial_{x_{1}} \mathrm{u}_{3} \\
\partial_{x_{1}} \mathrm{t}_{1}+\rho \omega^{2} \mathrm{u}_{3}
\end{array}\right)
$$

175 with

$$
\sigma_{11}(\mathbf{Y})=2 \mu(1+\alpha) \partial_{x_{1}} \mathbf{u}_{1}-\alpha \mathbf{t}_{3}, \quad \alpha=\lambda /(\lambda+2 \mu) .
$$

The guided modes are obtained by searching solutions to problem (6) in the form

$$
\left(\begin{array}{l}
\mathbf{X}(x) \\
\mathbf{Y}(x)
\end{array}\right)=\left(\begin{array}{c}
\mathcal{X}\left(x_{1}\right) \\
\mathcal{Y}\left(x_{1}\right)
\end{array}\right) e^{i \beta x_{3}}
$$

with $\beta \in \mathbb{C}$, which amounts to

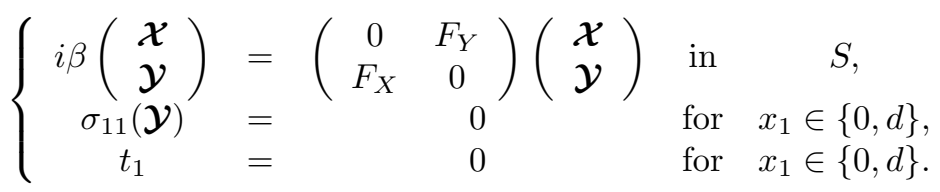


By using the spectral properties of the operators $F_{X}$ and $F_{Y}$ (see [30]) we obtain a discrete family of guided modes depending on $n \in \mathbb{N}$, given either in the $\mathbf{X}, \mathbf{Y}$ variables or in the $\mathbf{u}$ variable, by:

$$
\left(\begin{array}{c}
\mathbf{X}_{n}^{ \pm}(x) \\
\mathbf{Y}_{n}^{ \pm}(x)
\end{array}\right)=\left(\begin{array}{c} 
\pm \mathcal{X}_{n}\left(x_{1}\right) \\
\mathcal{Y}_{n}\left(x_{1}\right)
\end{array}\right) e^{ \pm i \beta_{n} x_{3}}, \quad \mathbf{u}_{n}^{ \pm}(x)=\left(\begin{array}{c}
u_{1}^{n}\left(x_{1}\right) \\
\pm u_{3}^{n}\left(x_{1}\right)
\end{array}\right) e^{ \pm i \beta_{n} x_{3}}
$$

In $2 \mathrm{D}$, the guided modes defined above are also known in the literature as the Lamb modes. In practice, for some given $\omega$, the $\beta_{n}$ do not have an analytical expression. The dispersion relation that they satisfy, as well as the corresponding displacement $\left(u_{1}^{n}, u_{3}^{n}\right)$ are specified in appendix B. An essential result (see [30]) concerning these guided modes is the following biorthogonality relationship, for $m, n \in \mathbb{N}$ :

$$
\left(\mathcal{X}_{n} \mid \mathcal{Y}_{m}\right)_{S}=\delta_{n m} J_{n}
$$

for some constant $J_{n}$, where the bilinear form $(\cdot \mid \cdot)_{S}$ is defined for $\mathcal{X}=\left(t_{1}, u_{3}\right)^{T}$ and $\mathcal{Y}=\left(u_{1}, t_{3}\right)^{T}$ in $\mathbf{L}^{2}(S)=\left(L^{2}(S)\right)^{2}$ by

$$
(\boldsymbol{\mathcal { X }} \mid \mathcal{Y})_{S}=\int_{0}^{d}\left(\mathcal{X}_{1} \mathcal{Y}_{1}+\mathcal{X}_{3} \mathcal{Y}_{3}\right) d s=\int_{0}^{d}\left(t_{1} u_{1}+u_{3} t_{3}\right) d s
$$

The bilinear form (11) can be extended by duality to the case when $\mathcal{X} \in$ $\tilde{H}^{-\frac{1}{2}}(S) \times H^{\frac{1}{2}}(S)$ and $\mathcal{Y} \in H^{\frac{1}{2}}(S) \times \tilde{H}^{-\frac{1}{2}}(S)$, where $\tilde{H}^{-\frac{1}{2}}(S)$ is the dual space of $H^{\frac{1}{2}}(S)$. The following assumption is made in the sequel.

Assumption 1. The frequency $\omega$ is such that none of the $\beta_{n}$ vanishes and none of the elements of the family $\left(\mathcal{X}_{n}, \mathcal{Y}_{n}\right)$ satisfies $\left(\mathcal{X}_{n} \mid \mathcal{Y}_{n}\right)_{S}=0$.

Assumption 1 eliminates the possibility of infinite phase velocity and of vanishing group velocity (see [33]). Such assumption enables us to set $J_{n}=1$ for all $n \in \mathbb{N}$. From the numerical results, Assumption 1 seems to be violated for at most a countable set of frequencies $\omega$. The guided modes for which $\beta_{n}$ is purely real are said to be propagating, since they propagate at long distance without attenuation. The guided modes can then be organized in two families:

- the rightgoing modes $\left(\beta_{n}, \mathcal{X}_{n}, \mathcal{Y}_{n}\right)$ for $n \in \mathbb{N}$, which correspond to $\operatorname{Im}\left(\beta_{n}\right)>$ 0 (for non-propagating modes) or $\frac{\partial \omega}{\partial \beta_{n}}>0$ (for propagating modes), where $\omega$ is an implicit function of $\beta_{n}$ via the dispersion relation 60.

- the leftgoing modes $\left(-\beta_{n},-\mathcal{X}_{n}, \mathcal{Y}_{n}\right)$ for $n \in \mathbb{N}$.

It should be noted that the non-propagating modes are either evanescent $\left(\beta_{n}\right.$ is purely imaginary) or inhomogeneous ( $\beta_{n}$ is not purely imaginary). Without loss of generality, we assume that the propagating modes are ordered in such a way that the sequence of $\beta_{n}$ decreases with respect to $n$.

\subsection{The radiation condition}

Let us assume the following conjecture. 
Conjecture 2. For every $\mathcal{X} \in \tilde{H}^{-\frac{1}{2}}(S) \times H^{\frac{1}{2}}(S)$ we have

$$
\mathcal{X}=\sum_{n \in \mathbb{N}}\left(\mathcal{X} \mid \mathcal{Y}_{n}\right)_{S} \mathcal{X}_{n}
$$

for every $\mathcal{Y} \in H^{\frac{1}{2}}(S) \times \tilde{H}^{-\frac{1}{2}}(S)$ we have

$$
\mathcal{Y}=\sum_{n \in \mathbb{N}}\left(\mathcal{X}_{n} \mid \mathcal{Y}\right)_{S} \mathcal{Y}_{n}
$$

and there exists $C>0$ such that

$$
\begin{aligned}
& \sum_{n}\left|\left(\mathcal{X} \mid \mathcal{Y}_{n}\right)_{S}\right|^{2} \leq C\|\mathcal{X}\|_{\tilde{H}^{-\frac{1}{2}}(S) \times H^{\frac{1}{2}}(S)}^{2} \\
& \sum_{n}\left|\left(\mathcal{X}_{n} \mid \mathcal{Y}\right)_{S}\right|^{2} \leq C\|\mathcal{Y}\|_{H^{\frac{1}{2}}(S) \times \tilde{H}^{-\frac{1}{2}}(S)}^{2} .
\end{aligned}
$$

Decompositions (12) and 13) are also true for $\mathcal{X}$ and $\mathcal{Y}$ in $\mathbf{L}^{2}(S)$.

To our best knowledge, a rigorous proof of the above completeness result, which seems true according to all our numerical experiments, does not exist. Following [30, from conjecture 2 the family $\left(\beta_{n}, \mathcal{X}_{n}, \mathcal{Y}_{n}\right)$ enables us to define a continuous $\mathcal{Y}$-to- $\mathcal{X}$ operator $T$ acting on a transverse section $S$, precisely

$$
T:\left(\begin{array}{c}
H^{\frac{1}{2}}(S) \times \tilde{H}^{-\frac{1}{2}}(S) \rightarrow \tilde{H}^{-\frac{1}{2}}(S) \times H^{\frac{1}{2}}(S) \\
\mathcal{Y} \mapsto \sum_{n \in \mathbb{N}}\left(\mathcal{X}_{n} \mid \mathcal{Y}\right)_{S} \mathcal{X}_{n}
\end{array}\right) .
$$

It is shown in 30 that the radiation condition in the elastic waveguide can be imposed by restricting the scattering problem to a bounded domain $W_{R}$ located between a right transverse section $S_{R}=S \times\{R\}$ and a left transverse section $S_{-R}$ and by prescribing the boundary conditions $T_{+} \mathbf{Y}=\mathbf{X}$ on $S_{R}$ and $T_{-} \mathbf{Y}=-\mathbf{X}$ on $S_{-R}$, where $T_{+}$and $T_{-}$are acting on sections $S_{R}$ and $S_{-R}$, 225 respectively. In what follows, $\Gamma_{R}$ is the portion of $\Gamma$ delimited by the sections $S_{-R}$ and $S_{R}$.

\subsection{The fundamental solution}

The classical outgoing Green solution of the elastic waveguide $W$, denoted by $G_{u}^{\sigma}$, is such that for $y \in W_{R}$, the $2 \times 2$ tensor $G_{u}^{\sigma}(\cdot, y)$ satisfies the problem:

$$
\left\{\begin{array}{ccc}
-\operatorname{div} \sigma\left(G_{u}^{\sigma}(\cdot, y)\right)-\rho \omega^{2} G_{u}^{\sigma}(\cdot, y)=\delta(\cdot-y) \operatorname{Id}_{2} & \text { in } & W_{R} \\
\sigma\left(G_{u}^{\sigma}(\cdot, y)\right) \nu=0 & \text { on } & \Gamma_{R} \\
T_{ \pm} G_{Y}^{\sigma}(\cdot, y)= \pm G_{X}^{\sigma}(\cdot, y) & \text { on } & S_{ \pm R},
\end{array}\right.
$$

where $G_{X}^{\sigma}$ (resp. $G_{Y}^{\sigma}$ ) denotes the $2 \times 2$ tensors such that each line of $G_{X}^{\sigma}$ (resp. $G_{Y}^{\sigma}$ ) is formed by the $\mathbf{X}$ extension (resp. $\mathbf{Y}$ extension) of the corresponding line of tensor $G_{u}^{\sigma}$. By using the coordinates of $\mathcal{X}_{n}=\left(t_{1}^{n}, u_{3}^{n}\right)^{T}$ and $\mathcal{Y}_{n}=\left(u_{1}^{n}, t_{3}^{n}\right)^{T}$, the tensor $G_{u}^{\sigma}$ is given by

$$
G_{u}^{\sigma}(x, y)=-\sum_{n \in \mathbb{N}}\left(\begin{array}{cc}
u_{1}^{n}\left(x_{1}\right) u_{1}^{n}\left(y_{1}\right) & -s\left(x_{3}-y_{3}\right) u_{1}^{n}\left(x_{1}\right) u_{3}^{n}\left(y_{1}\right) \\
s\left(x_{3}-y_{3}\right) u_{3}^{n}\left(x_{1}\right) u_{1}^{n}\left(y_{1}\right) & -u_{3}^{n}\left(x_{1}\right) u_{3}^{n}\left(y_{1}\right)
\end{array}\right) \frac{e^{i \beta_{n}\left|x_{3}-y_{3}\right|}}{2}
$$


where $s$ is the sign function. As a useful tool to formulate the Linear Sampling

Method in our elastic waveguide, we introduce the extended outgoing Green tensor $G$ for the elastic waveguide already introduced in [1], which is well adapted to the $\mathbf{X}, \mathbf{Y}$ formalism. The $4 \times 4$ tensor $G$ is such that for $y \in W_{R}, G(\cdot, y)$ is the solution to the problem:

$$
\left\{\begin{array}{c}
\frac{\partial}{\partial x_{3}} G(\cdot, y)=\left(\begin{array}{cc}
0 & F_{Y} \\
F_{X} & 0
\end{array}\right) G(\cdot, y)-\delta(\cdot-y)\left(\begin{array}{cc}
\operatorname{Id}_{2} & 0_{2} \\
0_{2} & \operatorname{Id}_{2}
\end{array}\right) \\
\sigma_{11}\left(G_{Y}(\cdot, y)\right)=0, \quad \mathrm{t}_{1}\left(G_{Y}(\cdot, y)\right)=0 \\
T_{ \pm} G_{Y}(\cdot, y)= \pm G_{X}(\cdot, y)
\end{array}\right.
$$

Here, $G_{X}$ and $G_{Y}$ denote the $X$-rows and $Y$-rows of the matrix $G$, respectively. If we decompose the tensor $G$ into four $2 \times 2$ blocks, that is

$$
G=\left(\begin{array}{cc}
G_{X}^{X} & G_{X}^{Y} \\
G_{Y}^{X} & G_{Y}^{Y}
\end{array}\right)
$$

it is proved in [1] that these blocks can be specified as

$$
\begin{gathered}
G_{X}^{X}(x, y)=-\sum_{n \in \mathbb{N}} s\left(x_{3}-y_{3}\right) \mathcal{X}_{n}\left(x_{1}\right) \otimes \mathcal{Y}_{n}\left(y_{1}\right) \frac{e^{i \beta_{n}\left|x_{3}-y_{3}\right|}}{2}, \\
G_{X}^{Y}(x, y)=-\sum_{n \in \mathbb{N}} \mathcal{X}_{n}\left(x_{1}\right) \otimes \mathcal{X}_{n}\left(y_{1}\right) \frac{e^{i \beta_{n}\left|x_{3}-y_{3}\right|}}{2} \\
G_{Y}^{X}(x, y)=-\sum_{n \in \mathbb{N}} \mathcal{Y}_{n}\left(x_{1}\right) \otimes \mathcal{Y}_{n}\left(y_{1}\right) \frac{e^{i \beta_{n}\left|x_{3}-y_{3}\right|}}{2} \\
G_{Y}^{X}(x, y)=-\sum_{n \in \mathbb{N}} s\left(x_{3}-y_{3}\right) \mathcal{Y}_{n}\left(x_{1}\right) \otimes \mathcal{X}_{n}\left(y_{1}\right) \frac{e^{i \beta_{n}\left|x_{3}-y_{3}\right|}}{2} .
\end{gathered}
$$

By selecting among the rows of matrix $G$ those which correspond to the components of $\mathbf{u}$ and by selecting among the columns of $G$ those which correspond to the components of $\sigma \mathbf{e}_{3}$ (we recall that $\sigma \mathbf{e}_{3}=\left(\mathrm{t}_{1},-\mathrm{t}_{3}\right)^{T}$ ), we obtain the classical Green tensor $G_{u}^{\sigma}$ from the extended one. More generally and for simplicity, $G_{a}^{b}$ will denote the tensor obtained from $G$ by selecting among the rows of matrix $G$ those which correspond to the components of type $a$ and by selecting among the columns of $G$ those which correspond to the components of type $b$.

\section{The modal formulation of the Linear Sampling Method}

We briefly summarize the modal formulation of the Linear Sampling Method such as described in [1] for rigid obstacles. But the justification can be easily adapted to the case of voids or cracks (see [2]). As recalled in the introduction, the method itself is independent of the nature of the defect. 


\subsection{The theoretical foundation}

255 We consider the following scattering forward problem : for $\mathbf{f} \in\left(H^{-\frac{1}{2}}(\partial D)\right)^{2}$, find $\mathbf{u} \in\left(H^{1}\left(\Omega_{R}\right)\right)^{2}$ such that

$$
\left\{\begin{array}{ccc}
\operatorname{div} \sigma(\mathbf{u})+\rho \omega^{2} \mathbf{u}=0 & \text { in } & \Omega_{R} \\
\sigma(\mathbf{u}) \boldsymbol{\nu}=0 & \text { on } & \Gamma_{R} \\
\mathbf{u}=\mathbf{f} & \text { on } & \partial D \\
T_{ \pm} \mathbf{Y}= \pm \mathbf{X} & \text { on } & S_{ \pm R}
\end{array}\right.
$$

where $\Omega_{R}=W_{R} \backslash \bar{D}$. Let us denote by $u_{Y}^{s}(\cdot, y)$ the solution to problem 19 which corresponds to $f=-\left.G_{u}^{Y}(\cdot, y)\right|_{\partial D}$ for $y \in \hat{S}:=S_{-R} \cup S_{R}$ and by $X_{Y}^{s}(\cdot, y)$ the $\mathbf{X}$ extension of $u_{Y}^{s}(\cdot, y)$. Similarly, let us denote by $u_{X}^{s}(\cdot, y)$ the solution to problem 190 which corresponds to $f=-\left.G_{u}^{X}(\cdot, y)\right|_{\partial D}$ for $y \in \hat{S}$ and by $Y_{X}^{s}(\cdot, y)$ the $\mathbf{Y}$ extension of $u_{X}^{s}(\cdot, y)$. We consider the inverse problem of finding the obstacle $D$ from the trace on $\hat{S}$ of either $X_{Y}^{s}(\cdot, y)$ or $Y_{X}^{s}(\cdot, y)$, for all $y \in \hat{S}$. In order to solve it we introduce the near field operators $N_{X}$ and $N_{Y}$ defined by

$$
N_{X}:\left(\begin{array}{c}
\mathbf{L}^{2}(\hat{S}) \rightarrow \mathbf{L}^{2}(\hat{S}) \\
\mathbf{h} \mapsto \int_{\hat{S}} X_{Y}^{s}(x, y) \mathbf{h}(y) d s(y), \quad x \in \hat{S}
\end{array}\right)
$$

and

$$
N_{Y}:\left(\begin{array}{c}
\mathbf{L}^{2}(\hat{S}) \rightarrow \mathbf{L}^{2}(\hat{S}) \\
\mathbf{h} \mapsto \int_{\hat{S}} Y_{X}^{s}(x, y) \mathbf{h}(y) d s(y), \quad x \in \hat{S}
\end{array}\right) .
$$

${ }_{265}$ The Linear Sampling Method relies on the following theorem (see Theorem 3.6 in [1]). The proof of such theorem follows exactly the same lines as the proof of Theorem 2 in [34] by using the factorization of the operators $N_{X}$ and $N_{Y}$ which is derived and analyzed in $[1$.

Theorem 3. Let $N_{X}$ and $N_{Y}$ be the near field operators defined by (20) and 270 (21) and $\mathbf{p}$ be some unit vector in $\mathbb{R}^{2}$. Except maybe for a countable set of frequencies $\omega$, the following statements hold:

- if $z \in D$, for all $\varepsilon>0$ there exists some field $\mathbf{h}_{\varepsilon}(\cdot, z, \mathbf{p}) \in \mathbf{L}^{2}(\hat{S})$ such that

$$
\left\|N_{X} \mathbf{h}_{\varepsilon}-G_{X}^{Y}(\cdot, z) \mathbf{p}\right\|_{\mathbf{L}^{2}(\hat{S})} \leq \varepsilon
$$

and some field $\mathbf{h}_{\varepsilon}(\cdot, z, \mathbf{p}) \in \mathbf{L}^{2}(\hat{S})$ such that

$$
\left\|N_{Y} \mathbf{h}_{\varepsilon}-G_{Y}^{X}(\cdot, z) \mathbf{p}\right\|_{\mathbf{L}^{2}(\hat{S})} \leq \varepsilon
$$

In these two cases, for a given $\varepsilon>0$,

$$
\lim _{z \rightarrow \partial D}\left\|\mathbf{h}_{\varepsilon}(\cdot, z, \mathbf{p})\right\|_{\mathbf{L}^{2}(\hat{S})}=+\infty .
$$


- if $z \in \Omega_{R}=W_{R} \backslash \bar{D}$, for all field $\mathbf{h}_{\varepsilon}(\cdot, z, \mathbf{p}) \in \mathbf{L}^{2}(\hat{S})$ such that

$$
\left\|N_{X} \mathbf{h}_{\varepsilon}-G_{X}^{Y}(\cdot, z) \mathbf{p}\right\|_{\mathbf{L}^{2}(\hat{S})} \leq \varepsilon \quad \text { or } \quad\left\|N_{Y} \mathbf{h}_{\varepsilon}-G_{Y}^{X}(\cdot, z) \mathbf{p}\right\|_{\mathbf{L}^{2}(\hat{S})} \leq \varepsilon,
$$

we have

$$
\lim _{\varepsilon \rightarrow 0}\left\|\mathbf{h}_{\varepsilon}(\cdot, z, \mathbf{p})\right\|_{\mathbf{L}^{2}(\hat{S})}=+\infty .
$$

Then a practical method to identify $D$ from one of the operators $N_{X}$ and $N_{Y}$ and given a unit vector $\mathbf{p}$, consists for all $z$ in some sampling grid, to solve in $\mathbf{L}^{2}(\hat{S})$ the equation

$$
N_{X} \mathbf{h}=\left.G_{X}^{Y}(\cdot, z)\right|_{\hat{S}} \mathbf{p}
$$

$$
N_{Y} \mathbf{h}=\left.G_{Y}^{X}(\cdot, z)\right|_{\hat{S}} \mathbf{p}
$$

and then to plot the function $\psi(z)=1 /\|\mathbf{h}(z)\|_{\mathbf{L}^{2}(\hat{S})}$, which from the above theorem turns out to be an indicator function of the defect.

\subsection{The modal formulation}

Now let us introduce the modal formulation of the LSM. To this end, let us denote by $\mathbf{u}_{n}^{s \pm}$ the solution to problem $\sqrt{19}$ which corresponds to $\mathbf{f}=-\left.\mathbf{u}_{n}^{ \pm}\right|_{\partial D}$, where $\mathbf{u}_{n}^{ \pm}$are the guided modes given for $n \in \mathbb{N}$ by $(10)$, and let us denote by $\mathbf{X}_{n}^{s \pm}$ the $\mathbf{X}$ extension of $\mathbf{u}_{n}^{s \pm}$. By our conjecture 2, we can decompose

$$
\begin{gathered}
\left.\mathbf{X}_{n}^{s+}\right|_{S_{-R}}=-\sum_{m \in \mathbb{N}} S_{m n}^{+-} \mathcal{X}_{m},\left.\quad \mathbf{X}_{n}^{s-}\right|_{S_{-R}}=-\sum_{m \in \mathbb{N}} S_{m n}^{--} \mathcal{X}_{m}, \\
\left.\mathbf{X}_{n}^{s+}\right|_{S_{R}}=\sum_{m \in \mathbb{N}} S_{m n}^{++} \boldsymbol{\mathcal { X }}_{m},\left.\quad \mathbf{X}_{n}^{s-}\right|_{S_{R}}=\sum_{m \in \mathbb{N}} S_{m n}^{-+} \boldsymbol{\mathcal { X }}_{m} .
\end{gathered}
$$

Similarly, we set $\mathbf{h}=\left(\mathbf{h}_{-}, \mathbf{h}_{+}\right) \in \mathbf{L}^{2}\left(S_{-R}\right) \times \mathbf{L}^{2}\left(S_{R}\right)$ with

$$
\mathbf{h}_{-}=-\sum_{n \in \mathbb{N}} h_{n}^{-} \mathcal{Y}_{n}, \quad \mathbf{h}_{+}=\sum_{n \in \mathbb{N}} h_{n}^{+} \mathcal{Y}_{n} .
$$

It is proved in [1] that the equation 22 is equivalent to the infinite system

$$
\left\{\begin{array}{l}
\sum_{n \in \mathbb{N}} e^{i \beta_{n} R}\left(S_{m n}^{+-} h_{n}^{-}+S_{m n}^{--} h_{n}^{+}\right)=e^{i \beta_{m}\left(R+z_{3}\right)}\left(\boldsymbol{\mathcal { X }}_{m}\left(z_{1}\right) \cdot \mathbf{p}\right) \\
\sum_{n \in \mathbb{N}} e^{i \beta_{n} R}\left(S_{m n}^{++} h_{n}^{-}+S_{m n}^{-+} h_{n}^{+}\right)=-e^{i \beta_{m}\left(R-z_{3}\right)}\left(\boldsymbol{\mathcal { X }}_{m}\left(z_{1}\right) \cdot \mathbf{p}\right)
\end{array} \quad \forall m \in \mathbb{N} .\right.
$$

From (24) and 25), we can deduce that

$$
\begin{aligned}
& \left.\mathbf{Y}_{n}^{s+}\right|_{S_{-R}}=\sum_{m \in \mathbb{N}} S_{m n}^{+-} \mathcal{Y}_{m},\left.\quad \mathbf{Y}_{n}^{s-}\right|_{S_{-R}}=\sum_{m \in \mathbb{N}} S_{m n}^{--} \mathcal{Y}_{m}, \\
& \left.\mathbf{Y}_{n}^{s+}\right|_{S_{R}}=\sum_{m \in \mathbb{N}} S_{m n}^{++} \mathcal{Y}_{m},\left.\quad \mathbf{Y}_{n}^{s-}\right|_{S_{R}}=\sum_{m \in \mathbb{N}} S_{m n}^{-+} \mathcal{Y}_{m},
\end{aligned}
$$


so that by choosing now

$$
\mathbf{h}_{-}=\sum_{n \in \mathbb{N}} h_{n}^{-} \boldsymbol{\mathcal { X }}_{n}, \quad \mathbf{h}_{+}=\sum_{n \in \mathbb{N}} h_{n}^{+} \boldsymbol{\mathcal { X }}_{n}
$$

we can prove similarly that the equation 23 is equivalent to the infinite system

295

$$
\left\{\begin{array}{l}
\sum_{n \in \mathbb{N}} e^{i \beta_{n} R}\left(S_{m n}^{+-} h_{n}^{-}+S_{m n}^{--} h_{n}^{+}\right)=e^{i \beta_{m}\left(R+z_{3}\right)}\left(\mathcal{Y}_{m}\left(z_{1}\right) \cdot \mathbf{p}\right) \\
\sum_{n \in \mathbb{N}} e^{i \beta_{n} R}\left(S_{m n}^{++} h_{n}^{-}+S_{m n}^{-+} h_{n}^{+}\right)=e^{i \beta_{m}\left(R-z_{3}\right)}\left(\mathcal{Y}_{m}\left(z_{1}\right) \cdot \mathbf{p}\right)
\end{array} \quad \forall m \in \mathbb{N} .\right.
$$

Like in acoustics, the above equivalence between the equation (22) (resp. the equation (23) and the system (26) (resp. the system (27)) shows that it is equivalent:

- to know the $\mathbf{X}$ (resp. $\mathbf{Y}$ ) extension of the scattered fields on $\hat{S}$ associated to all point sources $G_{u}^{Y}(\cdot, y)$ (resp. $\left.G_{u}^{X}(\cdot, y)\right)$ for $y \in \hat{S}$

- to know the projections on the $\mathcal{X}_{m}$ (resp. $\mathcal{Y}_{m}$ ) functions of the $\mathbf{X}$ (resp. $\mathbf{Y}$ ) extension of the scattered fields on $\hat{S}$ associated to all the guided modes $\mathbf{u}_{n}^{ \pm}$for $m, n \in \mathbb{N}$.

However, the notion of polarization is new in elasticity compared to acoustics: whing a single system is solved in acoustics (see [18), four differents systems can be solved in 2D elasticity, because of the scalar products $\left(\boldsymbol{\mathcal { X }}_{m} \cdot \mathbf{p}\right)$ and $\left(\mathcal{Y}_{m} \cdot \mathbf{p}\right)$ for $\mathbf{p}=(1,0)$ and $\mathbf{p}=(0,1)$. Since $\mathcal{X}=\left(t_{1}, u_{3}\right)^{T}$ and $\mathcal{Y}=\left(u_{1}, t_{3}\right)^{T}$, we obtain four different polarizations : $u_{1}, u_{3}, t_{1}$ and $t_{3}$.

Remark 4. We have shown in [2] that when the defect is a Neumann crack instead of a void, it can be detected point by point by using polarizations $t_{1}$ and $t_{3}$. More precisely, the correct polarization is $t_{1} \nu_{1}-t_{3} \nu_{3}$, where $\left(\nu_{1}, \nu_{3}\right)$ is the local normal to the crack. Such normal can be obtained locally by an optimization process.

\subsection{The regularization}

As proved in 1, 2, the near field operators $N_{X}$ and $N_{Y}$ are compact, so that the equations 222 and $(23)$ are ill-posed. A convenient way to regularize the infinite systems (26) and (27) is then to restrict the indices $m$ and $n$ to the $N$ first terms, where $N$ is the finite number of propagating modes. Indeed, the evanescent and inhomogeneous modes do not contribute to the scattered field

320 at a long distance from the defect, in particular on $\hat{S}=S_{-R} \cup S_{R}$ when $R$ is large, only the propagating modes do. Let us specify such discretization for the system (27). We hence define the matrices and vectors

$$
\begin{gathered}
S^{- \pm}=\left(S_{m n}^{- \pm}\right), \quad S^{+ \pm}=\left(S_{m n}^{+ \pm}\right), \quad m, n=0, \ldots, N-1 \\
H^{ \pm}=\left(h_{m}^{ \pm}\right), \quad F_{Y, p}^{ \pm}=e^{i \beta_{m}\left(R \mp z_{3}\right)}\left(\mathcal{Y}_{m}\left(z_{1}\right) \cdot \mathbf{p}\right), \quad m=0, \ldots, N-1 .
\end{gathered}
$$


We also introduce the $N \times N$ diagonal matrix $K$ formed by the diagonal terms $325 e^{i \beta_{n} R}, n=0, \ldots, N-1$, as well as the global matrices and vectors

$\mathcal{S}=\left(\begin{array}{cc}S^{+-} & S^{--} \\ S^{++} & S^{-+}\end{array}\right), \mathcal{U}=\mathcal{S}\left(\begin{array}{cc}K & 0 \\ 0 & K\end{array}\right), H=\left(\begin{array}{c}H^{-} \\ H^{+}\end{array}\right), \mathcal{F}_{Y, p}=\left(\begin{array}{c}F_{Y, p}^{-} \\ F_{Y, p}^{+}\end{array}\right)$.

With those notations, the regularization applied to the infinite system (27) leads to the $2 N \times 2 N$ system

$$
\mathcal{U} H=\mathcal{F}_{Y, p} .
$$

We refer to the $2 N \times 2 N$ matrix $\mathcal{S}$ as the scattering matrix while we refer to the $2 N \times 2 N$ matrix $\mathcal{U}$ as the LSM matrix. In what follows it will be convenient

330 to introduce the matrices $U^{- \pm}=S^{- \pm} K, U^{+ \pm}=S^{+ \pm} K$. Obviously, we obtain from $(26)$ the same system as 28$]$, except that the right-hand side $\mathcal{F}_{Y, p}$ is replaced by some vector $\mathcal{F}_{X, p}$. In view of the experimental setup of last section 7. it is important to address the back-scattering case, that is the data of the inverse problem are supported by $S_{-R}$ instead of $\hat{S}=S_{-R} \cup S_{R}$. The inverse problem consists then in finding the obstacle $D$ from the trace on $S_{-R}$ of either $X_{Y}^{s}(\cdot, y)$ or $Y_{X}^{s}(\cdot, y)$, for all $y \in S_{-R}$. In the back-scattering situation, it is readily seen that the $2 N \times 2 N$ system 28 is replaced by the $N \times N$ system

$$
U^{+-} H^{-}=F_{Y, p}^{-} .
$$

Remark 5. Here we highlight the fact that the system (28) is both a discretization and a regularization of the ill-posed problem (23). The discretization con-

340 sists of a projection on the transverse modes $\boldsymbol{\mathcal { X }}_{n}$. The regularization consists in considering only the integers $n$ associated with the propagating modes (this is justified in [18] in the acoustic case). This regularization seems relevant for at least two reasons. Firstly, it is based on a physical argument (the nonpropagating modes vanish at long distance). Secondly, it leads to a very limited number of meaningful degrees of freedom. Such physical way to regularize the ill-posed problem would not be possible by handling the data directly in the time domain. In particular, this strongly motivates us to use the modal formulation and hence a multi-frequency approach rather than a time domain approach like in [25], as detailed in the next section. \\ 5. The case of surface data in the time domain}

In this section, we explain how to exploit the modal formulation of the Linear Sampling Method exposed in section 4 to tackle the realistic NDT problem of section 2 .

\subsection{From time domain to frequency domain data}

First of all, by taking the Fourier transform with respect to time $t$ in the system (1), that is

$$
\widehat{\mathbf{v}}(x, \omega)=\int_{\mathbb{R}} \mathbf{v}(x, t) e^{i \omega t} d t,
$$


then for some fixed frequency $\omega>0$ the solution $\mathbf{u}=\widehat{\mathbf{v}}(\cdot, \omega)$ satisfies the scattering problem in $\Omega_{R}$ :

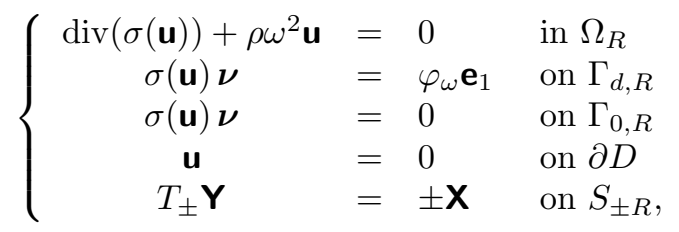

where $\Gamma_{d, R}=\Gamma_{d} \cap \Gamma_{R}, \Gamma_{0, R}=\Gamma_{0} \cap \Gamma_{R}$ and $\varphi_{\omega}=\widehat{\chi}(\omega) \varphi$.

Remark 6. It is fundamental to consider the exponential $e^{i \omega t}$ and not $e^{-i \omega t}$ in our definition (30) of the Fourier transform in order that $\mathbf{u}$ satisfies the radiation condition in problem (31). In the slightly simpler case of acoustics, this fact is fully justified in [29] with the help of the limiting absorption principle. Besides, the definition of (30) requires $\mathbf{v}$ to be sufficiently decreasing with respect to time $t$ at infinity. This aspect is discussed in the numerical section. In the presence of experimental data, the Fourier transform is of course replaced by the discrete Fourier transform.

For convenience, we also introduce the solution $\mathbf{u}^{i}$ to the system

$$
\left\{\begin{array}{clll}
\operatorname{div}\left(\sigma\left(\mathbf{u}^{i}\right)\right)+\rho \omega^{2} \mathbf{u}^{i} & = & & \text { in } W_{R} \\
\sigma\left(\mathbf{u}^{i}\right) \boldsymbol{\nu} & =\varphi_{\omega} \mathbf{e}_{1} & & \text { on } \Gamma_{d, R} \\
\sigma\left(\mathbf{u}^{i}\right) \boldsymbol{\nu} & = & & \text { on } \Gamma_{0, R} \\
T_{ \pm} \mathbf{Y}^{i} & = & &
\end{array}\right.
$$

As a result, $\mathbf{u}$ is the total field while $\mathbf{u}^{i}$ is the incident field and $\mathbf{u}^{s}=\mathbf{u}-\mathbf{u}^{i}$ is the scattered one. Besides, the scattered field $\mathbf{u}^{s}$ is solution to problem (19) with $\mathbf{f}=-\left.\mathbf{u}^{i}\right|_{\partial D}$. A convenient way to give an explicit expression of $\mathbf{u}^{i}$ is to use the fundamental solution of problem (32), which corresponds to the particular case when $\varphi_{\omega}$ is chosen as the Dirac distribution $\delta$ at point $y_{3}$. As proved in Appendix A, such fundamental solution coincides with $G_{u}^{t_{1}}$, which is obtained by selecting among the rows of matrix $G$ given by $(18)$ those which correspond to the components of $\mathbf{u}$ and by selecting the first column of $G$, that corresponds to the component $t_{1}$. The vector function $G^{t_{1}}$ is hence given by

$$
\left(\begin{array}{c}
G_{X}^{t_{1}} \\
G_{Y}^{t_{1}}
\end{array}\right)=-\sum_{n \in \mathbb{N}}\left(\begin{array}{c}
s\left(x_{3}-y_{3}\right) \mathcal{X}_{n}\left(x_{1}\right) \\
\mathcal{Y}_{n}\left(x_{1}\right)
\end{array}\right) \frac{u_{1}^{n}(d)}{2} e^{i \beta_{n}\left|x_{3}-y_{3}\right|}
$$

By convolution, it is readily seen that the incident field $\mathbf{u}^{i}$ in the extended variables $\mathbf{X}, \mathbf{Y}$ is expressed as

$$
\left(\begin{array}{c}
\mathbf{X}^{i} \\
\mathbf{Y}^{i}
\end{array}\right)=-\sum_{n \in \mathbb{N}} \int_{\mathbb{R}}\left(\begin{array}{c}
s\left(x_{3}-y_{3}\right) \mathcal{X}_{n}\left(x_{1}\right) \\
\mathcal{Y}_{n}\left(x_{1}\right)
\end{array}\right) \frac{u_{1}^{n}(d)}{2} e^{i \beta_{n}\left|x_{3}-y_{3}\right|} \varphi_{\omega}\left(y_{3}\right) d x_{3}
$$




\subsection{From surface to modal data}

We will need the following lemma.

Lemma 7. For $R^{\prime}>R$ and $\mathbf{g} \in H^{\frac{1}{2}}\left(S_{R}\right) \times \tilde{H}^{-\frac{1}{2}}\left(S_{R}\right)$, the extension $\mathbf{Y}$ of the solution $\mathbf{u}$ to the problem

$$
\left\{\begin{array}{clll}
\operatorname{div}(\sigma(\mathbf{u}))+\rho \omega^{2} \mathbf{u} & = & 0 & \text { in } S \times\left(R, R^{\prime}\right) \\
\sigma(\mathbf{u}) \boldsymbol{\nu} & = & 0 & \text { on }\left(\left\{x_{1}=d\right\} \cup\left\{x_{1}=0\right\}\right) \times\left(R, R^{\prime}\right) \\
\mathbf{Y} & =\mathbf{g} & \text { on } S_{R} \\
T_{+} \mathbf{Y} & =\mathbf{X} & \text { on } S_{R^{\prime}}
\end{array}\right.
$$

is given by

$$
\mathbf{Y}(x)=\sum_{n \in \mathbb{N}}\left(\mathcal{X}_{n} \mid \mathbf{g}\right)_{S_{R}} e^{i \beta_{n}\left(x_{3}-R\right)} \mathcal{Y}_{n}\left(x_{1}\right)
$$

Symmetrically, the extension $\mathbf{Y}$ of the solution $\mathbf{u}$ to the problem

$$
\left\{\begin{array}{clll}
\operatorname{div}(\sigma(\mathbf{u}))+\rho \omega^{2} \mathbf{u} & = & & \text { in } S \times\left(-R^{\prime},-R\right) \\
\sigma(\mathbf{u}) \boldsymbol{\nu} & = & & \text { on }\left(\left\{x_{1}=d\right\} \cup\left\{x_{1}=0\right\}\right) \times\left(-R^{\prime},-R\right) \\
\mathbf{Y} & =\mathbf{g} & \text { on } S_{-R} \\
T_{-} \mathbf{Y} & =-\mathbf{X} & \text { on } S_{-R^{\prime}}
\end{array}\right.
$$

is given by

$$
\mathbf{Y}(x)=\sum_{n \in \mathbb{N}}\left(\mathcal{X}_{n} \mid \mathbf{g}\right)_{S_{-R}} e^{-i \beta_{n}\left(x_{3}+R\right)} \mathcal{Y}_{n}\left(x_{1}\right)
$$

Proof. Let us begin with the first case (34). From Lemma 2.8 in 1, the solution to the first two common equations of systems (34) and 36 is given by

$$
\left(\begin{array}{l}
\mathbf{X}(x) \\
\mathbf{Y}(x)
\end{array}\right)=\sum_{n \in \mathbb{N}} a_{n}^{+}\left(\begin{array}{c}
\mathbf{X}_{n}^{+}(x) \\
\mathbf{Y}_{n}^{+}(x)
\end{array}\right)+a_{n}^{-}\left(\begin{array}{c}
\mathbf{X}_{n}^{-}(x) \\
\mathbf{Y}_{n}^{-}(x)
\end{array}\right)
$$

for some complex numbers $a_{n}^{+}$and $a_{n}^{-}$. Given the expression 10 of the guided modes $\mathbf{X}_{n}^{ \pm}, \mathbf{Y}_{n}^{ \pm}$, we obtain in particular

$$
\mathbf{X}(x)=\sum_{n \in \mathbb{N}}\left(a_{n}^{+} e^{i \beta_{n} x_{3}}-a_{n}^{-} e^{-i \beta_{n} x_{3}}\right) \mathcal{X}_{n}\left(x_{1}\right)
$$

and

$$
\mathbf{Y}(x)=\sum_{n \in \mathbb{N}}\left(a_{n}^{+} e^{i \beta_{n} x_{3}}+a_{n}^{-} e^{-i \beta_{n} x_{3}}\right) \mathcal{Y}_{n}\left(x_{1}\right) .
$$

From the definition (14) of the operator $T$, we have $T \mathcal{Y}_{n}=\mathcal{X}_{n}$ for all $n$, then the radiation condition on $S_{R}^{\prime}$ implies

$$
\sum_{n \in \mathbb{N}}\left(a_{n}^{+} e^{i \beta_{n} R^{\prime}}+a_{n}^{-} e^{-i \beta_{n} R^{\prime}}\right) \mathcal{X}_{n}\left(x_{1}\right)=\sum_{n \in \mathbb{N}}\left(a_{n}^{+} e^{i \beta_{n} R^{\prime}}-a_{n}^{-} e^{-i \beta_{n} R^{\prime}}\right) \mathcal{X}_{n}\left(x_{1}\right)
$$



is

$$
\mathbf{Y}(x)=\sum_{n \in \mathbb{N}} a_{n}^{+} e^{i \beta_{n} R} \mathcal{Y}_{n}\left(x_{1}\right)=\mathbf{g}(x),
$$

which implies by using the biorthogonality relationship (11) that

$$
a_{n}^{+} e^{i \beta_{n} R}=\left(\mathcal{X}_{n} \mid \mathbf{g}\right)_{S_{R}}
$$

for all $n$, and we end up with 35 . Let us now consider the other case $(36)$. The radiation condition on $S_{-R^{\prime}}$ now gives

$$
\sum_{n \in \mathbb{N}}\left(a_{n}^{+} e^{-i \beta_{n} R^{\prime}}+a_{n}^{-} e^{i \beta_{n} R^{\prime}}\right) \mathcal{X}_{n}\left(x_{1}\right)=-\sum_{n \in \mathbb{N}}\left(a_{n}^{+} e^{-i \beta_{n} R^{\prime}}-a_{n}^{-} e^{i \beta_{n} R^{\prime}}\right) \mathcal{X}_{n}\left(x_{1}\right)
$$

400

that is $a_{n}^{+}=0$ for all $n \in \mathbb{N}$. The boundary condition on $S_{-R}$ implies that

$$
\mathbf{Y}(x)=\sum_{n \in \mathbb{N}} a_{n}^{-} e^{i \beta_{n} R} \mathcal{Y}_{n}\left(x_{1}\right)=\mathbf{g}(x),
$$

so that

$$
a_{n}^{-} e^{i \beta_{n} R}=\left(\mathcal{X}_{n} \mid \mathbf{g}\right)_{S_{-R}}
$$

for all $n$, and we end up with 37 .

We have now to consider four configurations separately, depending on the fact that the source or the receiver is on the left or on the right of the defect.

Let us first consider a left source located at point $\left(d, y_{3}^{s-}\right)$ with $y_{3}^{s-}=-R-s \delta$, for $s=0, \ldots, M-1$. From $(33)$, the incident field is

$$
\begin{gathered}
\left(\begin{array}{c}
\mathbf{X}^{i}(x) \\
\mathbf{Y}^{i}(x)
\end{array}\right)=-\sum_{n \in \mathbb{N}}\left(\begin{array}{c}
\mathcal{X}_{n}\left(x_{1}\right) \\
\mathcal{Y}_{n}\left(x_{1}\right)
\end{array}\right) \frac{u_{1}^{n}(d)}{2} \int_{\mathbb{R}} e^{i \beta_{n}\left(x_{3}-y_{3}\right)} \varphi_{\omega}\left(y_{3}\right) d y_{3} \\
=-\sum_{n \in \mathbb{N}}\left(\begin{array}{l}
\mathbf{X}_{n}^{+}(x) \\
\mathbf{Y}_{n}^{+}(x)
\end{array}\right) \frac{u_{1}^{n}(d)}{2} \int_{\mathbb{R}} e^{-i \beta_{n} y_{3}} \varphi_{\omega}\left(y_{3}\right) d y_{3}
\end{gathered}
$$

The corresponding scattered field is then

$$
\left(\begin{array}{l}
\mathbf{X}^{s}(x) \\
\mathbf{Y}^{s}(x)
\end{array}\right)=-\sum_{n \in \mathbb{N}}\left(\begin{array}{c}
\mathbf{X}_{n}^{s+}(x) \\
\mathbf{Y}_{n}^{s+}(x)
\end{array}\right) \frac{u_{1}^{n}(d)}{2} \int_{\mathbb{R}} e^{-i \beta_{n} y_{3}} \varphi_{\omega}\left(y_{3}\right) d y_{3}
$$

where we recall that $\mathbf{X}_{n}^{s+}, \mathbf{Y}_{n}^{s+}$ are the $\mathbf{X}, \mathbf{Y}$ extensions of $\mathbf{u}_{n}^{s \pm}$, which is itself 410 the solution to problem (19) associated with $\mathbf{f}=-\left.\mathbf{u}_{n}^{ \pm}\right|_{\partial D}$. Since $\varphi_{\omega}\left(y_{3}\right)=$ $f_{\omega}\left(y_{3}-y_{3}^{s-}\right)$, where $f_{\omega}=\widehat{\chi}(\omega) f$, and taking into account the fact that $f$ is an even function, we obtain

$$
\mathbf{Y}^{s}(x)=-\sum_{n \in \mathbb{N}} \mathbf{Y}_{n}^{s+}(x) \frac{u_{1}^{n}(d)}{2} e^{i \beta_{n}(R+s \delta)} \int_{\mathbb{R}} e^{i \beta_{n} z} f_{\omega}(z) d z
$$


Let us now consider a reception point $\left(d, x_{3}\right)$ located on the right of the waveguide. By using Lemma 7 in the case of (34) and given the definition of the scattering matrix $S^{++}$, we have that

$$
\mathbf{Y}_{n}^{s+}\left(d, x_{3}\right)=\sum_{m \in \mathbb{N}}\left(\mathcal{X}_{m} \mid \mathbf{Y}_{n}^{s+}\right)_{S_{R}} e^{i \beta_{m}\left(x_{3}-R\right)} \mathcal{Y}_{m}(d)=\sum_{m \in \mathbb{N}} e^{i \beta_{m}\left(x_{3}-R\right)} S_{m n}^{++} \mathcal{Y}_{m}(d)
$$

By plugging this identity in the expression of $\mathbf{Y}^{s}$, we obtain

$$
\mathbf{Y}^{s}\left(d, x_{3}\right)=-\frac{\widehat{\chi}(\omega)}{2} \sum_{m, n \in \mathbb{N}} e^{i \beta_{m}\left(x_{3}-R\right)} S_{m n}^{++} u_{1}^{n}(d) e^{i \beta_{n}(R+s \delta)} f_{n} \mathcal{Y}_{m}(d),
$$

where

$$
f_{n}=\int_{\mathbb{R}} e^{i \beta_{n} z} f(z) d z .
$$

In view of (3), the Fourier transform of the measurement at the receiver located at $x_{3}^{r+}=R+r \delta$, for $r=0, \ldots, M-1$, is given by

$$
\widehat{\gamma}\left(x_{3}^{r+}, \omega\right)=\int_{\mathbb{R}} g\left(x_{3}-x_{3}^{r+}\right) \mathbf{u}_{1}^{s}\left(d, x_{3}\right) d x_{3},
$$

420

that is

$$
\widehat{\gamma}\left(x_{3}^{r+}, \omega\right)=-\frac{\widehat{\chi}(\omega)}{2} \sum_{m, n \in \mathbb{N}} e^{i \beta_{m} r \delta} g_{m} u_{1}^{m}(d) S_{m n}^{++} e^{i \beta_{n} R} u_{1}^{n}(d) f_{n} e^{i \beta_{n} s \delta},
$$

where

$$
g_{m}=\int_{\mathbb{R}} e^{i \beta_{m} z} g(z) d z .
$$

By restricting the two sums to the first $N$ terms, where $N$ is the number of propagating modes, we obtain that for $r, s=0, \ldots, M-1$, the measurement $M_{r s}^{++}$obtained at point $x_{3}^{r+}=R+r \delta$ for a source located at $y_{3}^{s-}=-R-s \delta$ is approximately given by

$$
M_{r s}^{++}=-\frac{\widehat{\chi}(\omega)}{2} \sum_{m, n=0}^{N-1} e^{i r \beta_{m} \delta} g_{m} u_{1}^{m}(d) U_{m n}^{++} u_{1}^{n}(d) f_{n} e^{i \beta_{n} s \delta}
$$

where we have used the fact that $U^{++}=S^{++} K$. Using matrices, we obtain that

$$
M^{++}=-\frac{\widehat{\chi}(\omega)}{2} R U^{++} E^{T},
$$

where

$$
R=V F_{g} T, \quad E=V F_{f} T
$$

and

- $F_{f}$ and $F_{g}$ are the $N \times N$ diagonal matrices formed by the diagonal terms $f_{n}$ and $g_{n}$, respectively, 
- $T$ is the $N \times N$ diagonal matrix formed by the diagonal terms $u_{1}^{n}(d)$,

- $V$ is the $M \times N$ Vandermonde matrix given by

$$
V_{m n}=e^{i m \beta_{n} \delta}, \quad m=0, \ldots, M-1, \quad n=0, \ldots, N-1 .
$$

In what follows, the matrices $F_{f}$ and $F_{g}$ will be called the source shape and receiver shape matrices, the matrix $T$ will be called the matrix of modes, while the matrices $R$ and $E$ will be called the reception and emission matrices.

Let us now consider a reception point $\left(d, x_{3}\right)$ located on the left of the waveguide. By using Lemma 7 in the case of (36) and by the definition of the scattering matrix $S^{+-}$, we have that

$$
\mathbf{Y}_{n}^{s+}\left(d, x_{3}\right)=\sum_{m \in \mathbb{N}}\left(\mathcal{X}_{m} \mid \mathbf{Y}_{n}^{s+}\right)_{S_{-R}} e^{-i \beta_{m}\left(x_{3}+R\right)} \mathcal{Y}_{m}(d)=\sum_{m \in \mathbb{N}} e^{-i \beta_{m}\left(x_{3}+R\right)} S_{m n}^{+-} \mathcal{Y}_{m}(d)
$$

For a source located at point $\left(d, y_{3}^{s-}\right)$, we hence obtain

$$
\mathbf{Y}^{s}\left(d, x_{3}\right)=-\frac{\widehat{\chi}(\omega)}{2} \sum_{m, n \in \mathbb{N}} e^{-i \beta_{m}\left(x_{3}+R\right)} S_{m n}^{+-} e^{i \beta_{n} R} u_{1}^{n}(d) e^{i \beta_{n} s \delta} f_{n} \mathcal{Y}_{m}(d)
$$

The Fourier transform of the measurement at the receiver located at $x_{3}^{r-}=$ $-R-r \delta$, for $r=0, \ldots, M-1$, is given by

$$
\widehat{\gamma}\left(x_{3}^{r-}, \omega\right)=-\frac{\widehat{\chi}(\omega)}{2} \sum_{m, n \in \mathbb{N}} e^{i \beta_{m} r \delta} g_{m} u_{1}^{m}(d) S_{m n}^{+-} e^{i \beta_{n} R} u_{1}^{n}(d) f_{n} e^{i \beta_{n} s \delta}
$$

so that for $r, s=0, \ldots, M-1$, the measurement $M_{r s}^{+-}$for a receiver located at $x_{3}^{r-}=-R-r \delta$ and for a source located at $x_{3}^{s-}=-R-s \delta$ is approximated by

$$
M_{r s}^{+-}=-\frac{\widehat{\chi}(\omega)}{2} \sum_{m, n=0}^{N-1} e^{i r \beta_{m} \delta} g_{m} u_{1}^{m}(d) U_{m n}^{+-} u_{1}^{n}(d) f_{n} e^{i \beta_{n} s \delta}
$$

${ }_{445}$ that is

$$
M^{+-}=-\frac{\widehat{\chi}(\omega)}{2} R U^{+-} E^{T} .
$$

Let us now consider a right source located at point $\left(d, y_{3}^{s+}\right)$ with $y_{3}^{s+}=R+s \delta$, for $s=0, \ldots, M-1$. From (33), the incident field is

$$
\begin{aligned}
\left(\begin{array}{c}
\mathbf{X}^{i}(x) \\
\mathbf{Y}^{i}(x)
\end{array}\right) & =-\sum_{n \in \mathbb{N}}\left(\begin{array}{c}
-\mathcal{X}_{n}\left(x_{1}\right) \\
\mathcal{Y}_{n}\left(x_{1}\right)
\end{array}\right) \frac{u_{1}^{n}(d)}{2} \int_{\mathbb{R}} e^{-i \beta_{n}\left(x_{3}-y_{3}\right)} \varphi_{\omega}\left(y_{3}\right) d y_{3} \\
& =-\sum_{n \in \mathbb{N}}\left(\begin{array}{c}
\mathbf{X}_{n}^{-}(x) \\
\mathbf{Y}_{n}^{-}(x)
\end{array}\right) \frac{u_{1}^{n}(d)}{2} \int_{\mathbb{R}} e^{i \beta_{n} y_{3}} \varphi_{\omega}\left(y_{3}\right) d y_{3}
\end{aligned}
$$


and the corresponding scattered field is then

$$
\left(\begin{array}{l}
\mathbf{X}^{s}(x) \\
\mathbf{Y}^{s}(x)
\end{array}\right)=-\sum_{n \in \mathbb{N}}\left(\begin{array}{c}
\mathbf{X}_{n}^{s-}(x) \\
\mathbf{Y}_{n}^{s-}(x)
\end{array}\right) \frac{u_{1}^{n}(d)}{2} \int_{\mathbb{R}} e^{i \beta_{n} y_{3}} \varphi_{\omega}\left(y_{3}\right) d y_{3}
$$

${ }_{450}$ By proceeding as previously, for $r, s=0, \ldots, M-1$, the measurement $M_{r s}^{-+}$for a receiver located at $x_{3}^{r+}=R+r \delta$ and for a source located at $y_{3}^{s+}=R+s \delta$ is approximated by $M_{r s}^{-+}$such that

$$
M^{-+}=-\frac{\widehat{\chi}(\omega)}{2} R U^{-+} E^{T}
$$

while the measurement $M_{r s}^{--}$for a receiver located at $x_{3}^{r-}=-R-r \delta$ and for a source located at $y_{3}^{s+}=R+s \delta$ is approximated by $M_{r s}^{--}$such that

$$
M^{--}=-\frac{\widehat{\chi}(\omega)}{2} R U^{--} E^{T}
$$

Gathering all the previous contributions (40, (43), (44) and 45, we end up with

$$
\mathcal{M}=-\frac{\hat{\chi}(\omega)}{2} \mathcal{R} \mathcal{U} \mathcal{E}^{T}
$$

where

$$
\mathcal{M}=\left(\begin{array}{cc}
M^{+-} & M^{--} \\
M^{++} & M^{-+}
\end{array}\right), \quad \mathcal{R}=\left(\begin{array}{cc}
R & 0 \\
0 & R
\end{array}\right), \quad \mathcal{E}=\left(\begin{array}{cc}
E & 0 \\
0 & E
\end{array}\right) .
$$

We hence conclude that for a given frequency, we can compute the LSM matrix $\mathcal{U}$ from the measurement matrix $\mathcal{M}$ by inverting the reception and emission ${ }_{460}$ matrices $\mathcal{R}$ and $\mathcal{E}$. In the case of back-scattering, the system to invert is reduced to 43 .

\subsection{The general strategy}

The global strategy to solve the inverse problem in the full-scattering situation consists in the following steps:

1. Compute or measure the transverse component of the scattered field $\mathbf{v}^{s}$ in the time domain at all receivers $x_{r}^{ \pm}$for all sources $x_{s}^{ \pm}$for $r, s=0, \ldots, M-1$.

2. Form the measurement matrix $\mathcal{M}(\omega)$ for all frequencies $\omega$.

3. Invert the system 46 to obtain the LSM Matrix $\mathcal{U}(\omega)$ for all $\omega$.

4. For all $z \in G$, where $G$ is a sampling grid, compute the indicator function $\psi(z, \omega)$ by inverting the system 28 .

5. Compute a global indicator function $\Psi$ of the defect as

$$
\Psi(z)=\left(\int_{\omega_{-}}^{\omega_{+}} \frac{\max _{z^{\prime} \in G}\left|\psi\left(z^{\prime}, \omega\right)\right|^{2}}{|\psi(z, \omega)|^{2}} d \omega\right)^{-1 / 2}
$$

for some lower and upper bounds $\omega_{-}$and $\omega_{+}$. 
In the back-scattering situation, we recall that the receivers and the sources are limited to $x_{r}^{-}$and $x_{s}^{-}$for $r, s=0, \ldots, M-1$, the measurement matrix reduces to $475 M^{+-}(\omega)$, the LSM matrix reduces to $U^{+-}(\omega)$ and is obtained by inverting the system (43), lastly the LSM system to invert for all point $z$ reduces to 290 . We also recall that the indicator functions $\Psi$ and $\psi(\cdot, \omega)$ are implicitly parametrized by one of the four polarizations $u_{1}, u_{3}, t_{1}$ and $t_{3}$. The definition of the global indicator function $\Psi$ given by (47) corresponds to a "serial" combination. Such 480 choice is discussed and justified in [26].

Remark 8. We point out that in general, the system (28) should be solved in the Tikhonov sense (like in [35]), since the underlying operator to invert is compact. But in our case, since the system (28) is already regularized (see Remark 5) and small, an additional regularization is not necessary when the amplitude of noise is reasonable.

\subsection{On the inversion of the emission and reception matrices}

A key point of our method is the computation of the LSM matrix $\mathcal{U}$ from the measurement matrix $\mathcal{M}$ given by (46), which amounts to inverting the reception and emission matrices $R$ and $E$. Given their definition (41), we are led to check the invertibility of the source and receiver shape matrices $F_{f}, F_{g}$, the matrix of modes $T$ and the Vandermonde matrix $V$.

\subsubsection{The source/receiver shape matrices}

Since the two matrices have the same form, we concentrate on the source shape matrix $F_{f}$, the diagonal term $f_{n}$ of which is given by (38). We choose

${ }_{495} f$ such that none of the $f_{n}$ vanishes for $n=0, \ldots, N-1$. In particular, if $f$ coincides with the Dirac function, $F_{f}$ is the identity matrix. We expect that, if the function $f$ is not "too far" from the Dirac, for example a hat function with a small support and a high amplitude, then $F_{f}$ will be invertible and well conditioned.

\subsubsection{The matrix of modes}

The matrix of modes $T$ is also diagonal, the diagonal term being given by $u_{1}^{n}(d)$ for $n=0, \ldots, N-1$, where $u_{1}^{n}(d)$ is the transverse component of the $n^{\text {th }}$ mode given by (10) on the upper boundary of the waveguide. The matrix $T$ is invertible if and only if none of the $u_{1}^{n}(d)$ vanishes for $n=0, \ldots, N-1$. It

${ }_{505}$ happens that there are some exceptional values of $\omega$ such that one of the $u_{1}^{n}(d)$ vanishes. To be more specific, we prove the following theorem in appendix B, where the notion of symmetric/antisymmetric mode is also defined.

Theorem 9. Let us assume that $\lambda>0$. The matrix $T$ is not invertible if and only if for some propagating mode $\left(\beta_{n}, \mathcal{X}_{n}, \mathcal{Y}_{n}\right), n=0, \ldots, N-1$, there exists

$(p, q) \in \mathbb{N}^{2}$ such that $p$ and $q$ are either both odd or both even, with

$$
\beta_{n}^{2}=\omega^{2} \frac{\rho}{\mu}-\left(\frac{p \pi-2 \kappa_{n}}{d}\right)^{2}=\omega^{2} \frac{\rho}{\lambda+2 \mu}-\left(\frac{q \pi-2 \kappa_{n}}{d}\right)^{2},
$$

where $\kappa_{n}=0$ if the mode is symmetric and $\kappa_{n}=\pi / 2$ if it is antisymmetric. 
We also prove in appendix $\mathrm{B}$ that for such mode, the corresponding $\beta_{n}$ is associated with both a symmetric and an antisymmetric mode. From a geometric point of view, this means that if we plot the dispersion curves associated with all the propagating guided modes in the plane $(\omega, \beta)$, the cases when $T$ is not invertible are those which correspond to the intersection of four curves: a curve associated with a symmetric mode, a curve associated with an antisymmetric mode and two curves of equations given by

$$
\beta^{2}=\omega^{2} \frac{\rho}{\mu}-\left(\frac{\pi m}{d}\right)^{2}
$$

and

$$
\beta^{2}=\omega^{2} \frac{\rho}{\lambda+2 \mu}-\left(\frac{\pi n}{d}\right)^{2},
$$

for $m, n \in \mathbb{N}$, respectively. In the figure 2 , above is represented the condition number of the matrix $T$ as a function of the frequency $\omega$, while below are represented the dispersion curves in the coordinates $(\omega, \beta)$, as well as the curves (48) and (49). Since $T$ is a diagonal matrix, the condition number is the ratio between the largest diagonal term and the lowest one with respect to the complex norm. The parameters $d, \rho, \lambda$ and $\mu$ are those which are chosen in the numerical section. We observe that the peaks in the condition number of $T$ coincide with the intersections of the four curves mentioned above.
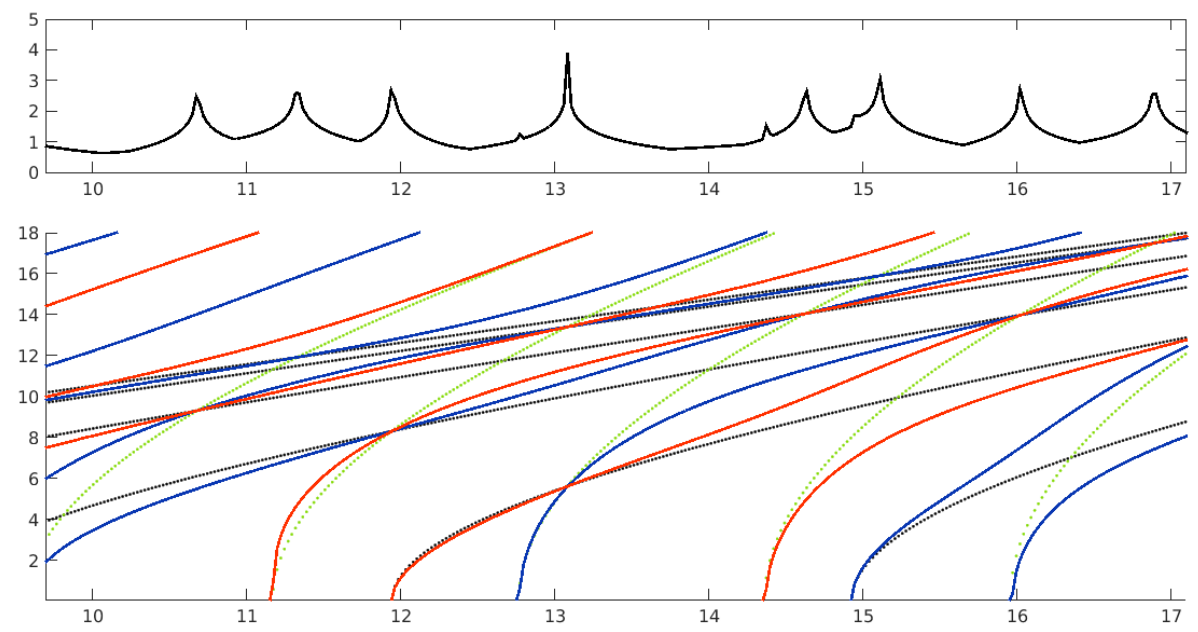

Figure 2: Above : logarithm of the condition number of the matrix $T$ as a function of $\omega$. Below : dispersion curves for symmetric modes (blue) and for antisymmetric modes (red), 48-type of curve (dashed green), 49 -type of curve (dashed black).

\subsubsection{The Vandermonde matrix}

The invertibility and the conditioning of the rectangular Vandermonde matrix $V$ given by $(42)$ have already been studied in the acoustic case in [26]. 
However, the elastic case is more complicated since the $\beta_{n}$ are not known analytically, contrary to the acoustic case. The matrix $V$ depends only on the $\beta_{n}$, on the number $M$ of sources and receivers and on the smallest distance $\delta$ between two sources or two receivers. It is recalled in the appendix A of [26] that for a Vandermonde matrix with entries on the unit circle, that is

$$
V_{m n}=e^{2 \pi i m f_{n}}, \quad m=0, \ldots, M-1, \quad n=0, \ldots, N-1,
$$

for real numbers $f_{n}$ and $M \geq N$, the matrix $V^{*} V$ is invertible if and only if for all $n^{\prime} \neq n, f_{n^{\prime}}-f_{n}$ is not an integer. Here

$$
f_{n}=\frac{\beta_{n} \delta}{2 \pi}, \quad n=0, \ldots, N-1 .
$$

An important issue, which can be seen on the dispersion curves, is that we may have $\beta_{n}(\omega)=\beta_{n^{\prime}}(\omega)$ for $n \neq n^{\prime}$ for some exceptional values of $\omega$, that 540 is multiple eigenvalues. The matrix $V^{*} V$ is not invertible in that case. We hence assume from now on that $\omega$ is such that all the $\beta_{n}$ are different, which in particular implies that the matrix of modes $T$ is invertible. Since the $\beta_{n}$ are ordered decreasingly, the invertibility of $V^{*} V$ is achieved if the distance $\delta$ satisfies

$$
\delta<\frac{2 \pi}{\beta_{0}-\beta_{N-1}} .
$$

${ }_{545}$ Let us consider now the condition number $\kappa(V)$ of $V$, which is defined by

$$
\kappa(V)=\sqrt{\frac{\sigma_{\max }}{\sigma_{\min }}},
$$

where $\sigma_{\max }$ and $\sigma_{\min }$ are the largest and smallest eigenvalues of $V^{*} V$, respectively. Let us introduce the wrap-around distance on the unit interval, that is

$$
d_{w}(f, g)=\inf _{q \in \mathbb{Z}}|f-g+q|
$$

as well as the minimal separation between the $f_{n}$ as

$$
\Delta=\min _{\substack{n, n^{\prime}=0, \ldots, N-1 \\ n \neq n^{\prime}}} d_{w}\left(f_{n}, f_{n^{\prime}}\right) .
$$

The following theorem is proved in [36, 26].

Theorem 10. If the minimal separation $\Delta$ defined by (51) satisfies $M>1 / \Delta+$ 1 , then

$$
\kappa(V) \leq \sqrt{\frac{M+1 / \Delta-1}{M-1 / \Delta-1}} .
$$

Such theorem shows that $\kappa(V)$ is decreasing with respect to $M$ and with respect to $\Delta$. It is then tempting to use as many sources and receivers as possible. Maximizing $\Delta$ with respect to $\delta$ is desirable but contrary to the acoustic 
case, it is rather difficult in elasticity due to the fact that the $\beta_{n}$ are not known analytically. In the case when all the $\beta_{n}$ are positive, which means that there are no inverse modes (the phase velocity and the group velocity have the same sign), we simply choose

$$
\delta=\lambda_{0}:=\frac{2 \pi}{\beta_{0}},
$$

which fulfills $(50)$. In the acoustic case, it was proved in [26] that such choice of $\delta$ was the optimal one. In the figure 3 , we have represented the condition number of the Vandermonde matrix $V$ as a function of $\delta / \lambda_{0}$, for $P=12$ and for increasing values of $M$, that is $M=P, M=2 P, M=4 P$ and $M=8 P$. Such figure confirms that choosing $\delta$ as in (52) ensures the invertibility of $V^{*} V$ and 565 is a relevant value (the peaks correspond to the non-invertibility cases). It also confirms that the condition number improves when $M$ increases.

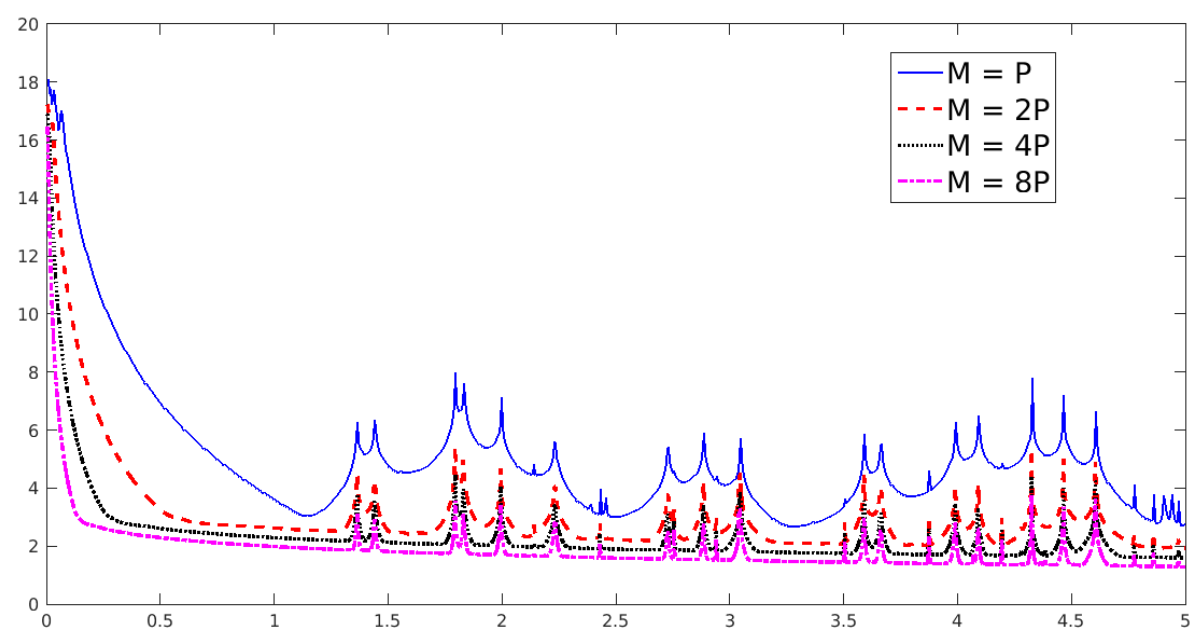

Figure 3: Logarithm of the condition number of the matrix $V$ as a function of $\delta / \lambda_{0}$, for $M=P, M=2 P, M=4 P$ and $M=8 P$.

\section{Numerical results}

In this section we test the strategy detailed in section 5.3 with the help of numerical experiments, not only for rigid obstacles, but also for voids and cracks. The height of the waveguide is $d=1 \mathrm{~mm}$ and the selected material constants are

$$
\lambda=121 \mathrm{GPa}, \quad \mu=80 \mathrm{GPa}, \quad \rho=7900 \mathrm{~kg} \cdot \mathrm{m}^{-3} .
$$

The artificial data are obtained by solving the forward problems (1) in the time domain. To this aim we use the code Echo, which is developed at CEA-List 37. It relies on a spectral finite element method in space (with domain decom575 position), a leap-frog scheme in time and Perfectly Matched Layers to bound 
the domain. We also need the computation of the guided modes $\left(\beta_{n}, \mathcal{X}_{n}, \mathcal{Y}_{n}\right)$, which is based on the SAFE method [33, 38, which allows us to solve a generalized eigenvalue problem with the help of a finite element method. The function $f$ in $(2)$ is chosen as

$$
f\left(x_{3}\right)=\frac{10}{d} \max \left(1-\frac{10\left|x_{3}\right|}{d}, 0\right)
$$

while the function $\chi$ is chosen as

$$
\chi(t)=\sum_{n \in \mathcal{I}} \chi_{n}(t)=\sum_{n \in \mathcal{I}}\left[\sin \left(a_{n} t\right) \exp \left(-b_{n}\left(t-c_{n}\right)^{2}\right)\right]^{\prime},
$$

with $\mathcal{I} \subset \mathbb{N}$. The shape of $\chi$ given by $(53)$ is designed in such a way that the support of $\hat{\chi}$ avoids all the cut-off frequencies $\omega_{n}$, which are the frequencies such that there exists a guided mode with vanishing group velocity, that is $\partial \omega / \partial \beta_{n}=0$. These are also the frequencies such that $\left(\mathcal{X}_{n} \mid \mathcal{Y}_{n}\right)_{S}=0$ (see assumption 17. As detailed in [26], this enables us to obtain some scattered fields which decay rapidly with respect to time and then to reasonably bound the duration of the forward computations. But contrary to acoustics, these special frequencies $\omega_{n}$ have to be computed and are not equally distributed. Once they have been computed, the constants $a_{n}, b_{n}$ and $c_{n}$ are set to

$$
\left\{\begin{aligned}
b_{n} & =\frac{\pi^{2}}{200 d^{2}}, \\
c_{n} & =\frac{5}{\sqrt{2 b_{n}}}, \\
\frac{a_{n}+\sqrt{a_{n}^{2}+16 b_{n}}}{2} & =\frac{1}{2}\left(\omega_{n}-\omega_{n-1}\right) .
\end{aligned}\right.
$$

Moreover, we choose the set $\mathcal{I}$ of $n \in \mathbb{N}$ such that the support of $\chi_{n}$ is sufficiently small. In practice we have set $\mathcal{I}=\{8,11,14\}$. This implies that for all the frequencies which belong to the support of $\hat{\chi}$, the corresponding number of propagating modes is 8,11 or 14 . The frequencies $\omega_{-}$and $\omega_{+}$in (47) are the lower and upper bounds of the support of $\widehat{\chi}$, that is $\omega_{-}=\omega_{7}$ and $\omega_{+}=\omega_{14}$. The function $g$ in (3) is simply chosen as the Dirac distribution, which corresponds to a pointwise measurement. The number of sources/receivers on each side of the waveguide is $M=42$ while the $x_{3}$ coordinate of the closest sensor is $R=1$ and the distance $\delta$ between two sources/receivers is given by 52 , with $\beta_{0}$ associated with the largest frequency $\omega$, that is $\omega_{+}$. The parameters $M, R$ and $\delta$ governing the location of sources/receivers (see (4)) are then determined. The sampling grid $G$ consists of the subpart of the waveguide delimited by $x_{3}=-R$ and $x_{3}=R$. In figure 4 we present some identifications results of an obstacle characterized by a Dirichlet boundary condition and formed by a square or two rectangles, either in the full-scattering situation or the back-scattering situation, with polarization $u_{1}$. We observe that the Dirichlet defects are well retrieved, in particular in the full-scattering situation, as expected. In figure 5 we present 

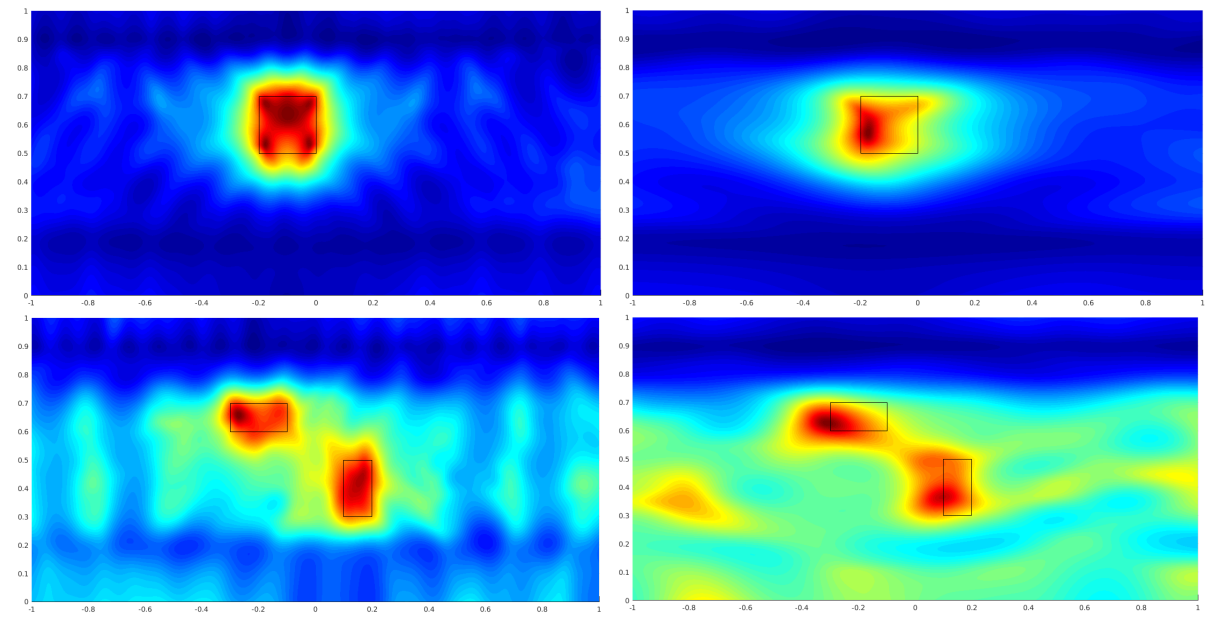

Figure 4: Obstacle with Dirichlet boundary condition. Top: one square. Bottom: two rectangles. Left: full-scattering situation. Right: back-scattering situation.

the same results for a square characterized by a Neumann boundary condition, in the full-scattering situation, and with two kinds of polarizations: $t_{1}$ and $t_{3}$. We observe that voids are not as well retrieved as Dirichlet defects, which is
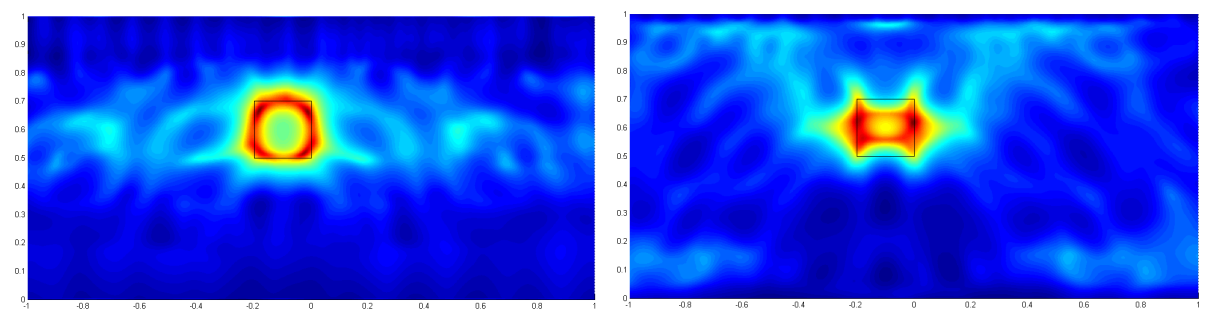

Figure 5: Obstacle with Neumann boundary condition in the full-scattering situation. Left: polarization $t_{1}$. Right: polarization $t_{3}$.

usual in sampling methods. Lastly, in figure 6 we present the same results for two straight cracks, in the full-scattering situation, and with polarizations $t_{1}$ and $t_{3}$. Those results are consistent with Remark 4 in the case of cracks, the polarization has to be in accordance with the local normal to the crack. The crack on the left is better retrieved with polarization $t_{1}$ while the crack on the ${ }_{615}$ right is better retrieved with polarization $t_{3}$. An optimization procedure to compute such local normal for curved cracks (not used here) is described in [2. 

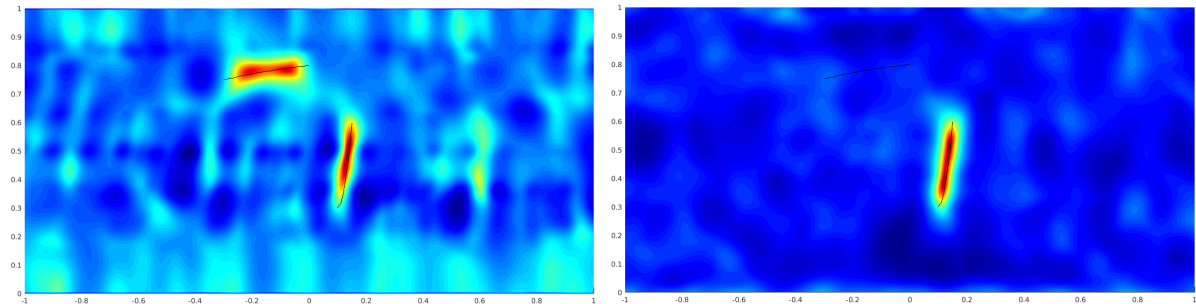

Figure 6: Case of two cracks in the full-scattering situation. Left: polarization $t_{1}$. Right: polarization $t_{3}$.

\section{Experimental results}

We complete this article with some experimental results on a steel plate of dimensions $12 \mathrm{~mm} \times 110 \mathrm{~mm} \times 500 \mathrm{~mm}$ in the $x_{1}, x_{2}$ and $x_{3}$ directions, respectively. As shown in figure 7 (on the left), the plate is divided into 3 parts in the $x_{2}$ direction:

- a zone of width $50 \mathrm{~mm}$ which doesn't contain any defect,

- a zone of width $10 \mathrm{~mm}$ which contains a cylindrical notch, the section of which is a rectangle of dimension $0.25 \mathrm{~mm}$ in the $x_{3}$ direction and $2 \mathrm{~mm}$ in the direction $x_{1}$,

- a zone of width $50 \mathrm{~mm}$ which contains a cylindrical hole, the section of which is a disc of diameter $2 \mathrm{~mm}$.

The zone which is free of defect enables us to measure the incident field while ${ }_{630}$ the two others enable us to measure the total fields in the presence of the defect. The material constants of the plate are completely determined by measurements of the 3 following quantities: the density $\rho$, the celerity of $\mathrm{P}$ waves $c_{P}$ and the celerity of $\mathrm{S}$ waves $c_{S}$, which in turn enable us to compute the Lamé constants $\lambda$ and $\mu$ thanks to

$$
c_{P}=\sqrt{\frac{\lambda+2 \mu}{\rho}}, \quad c_{S}=\sqrt{\frac{\mu}{\rho}} .
$$

We have found

$$
\rho=7926 \mathrm{~kg} \cdot \mathrm{m}^{-3}, \quad c_{P}=5897 \mathrm{~m} \cdot \mathrm{s}^{-1}, \quad c_{S}=3225 \mathrm{~m} \cdot \mathrm{s}^{-1} .
$$

A classical linear ultrasonic transducer array is used for the non-destructive inspection of the plate. More precisely, the mean frequency of that transducer is $2 \mathrm{Mhz}$ and its bandwidth contains frequencies which correspond to 12 to 25 propagating modes. It consists of 128 elements of size $18 \mathrm{~mm} \times 0.55 \mathrm{~mm}$ in ${ }_{640}$ the $x_{2}$ and $x_{3}$ directions, respectively, the distance between two elements being $0.25 \mathrm{~mm}$, which implies that the distance between two sources/receivers (see (4) ) is $\delta=0.8 \mathrm{~mm}$. As concerns the signal which is actually obtained with such 
transducer, the time function $\chi$ in (1) can be measured directly, while the space functions in (2) and (3) are both approximated by

$$
f\left(x_{3}\right)=g\left(x_{3}\right)=e^{-x_{3}^{2} / \sigma^{2}} \chi_{[-\ell / 2, \ell / 2]}\left(x_{3}\right),
$$

where $\ell$ is the size of the element in the $x_{3}$ direction, $\chi_{[-\ell / 2, \ell / 2]}$ is the indicator function of the segment $[-\ell / 2, \ell / 2]$ and $\sigma$ is calibrated from experimental observations. We have chosen $\ell / \sigma=1.1$. Note that the value of that ratio is not that sensitive. In the following experiments, only the back-scattering situation is tested. A photograph of the experimental setup is shown in figure 7 (on the right). As explained in the paragraph 5.4 .3 , the Vandermonde matrix $V$, and
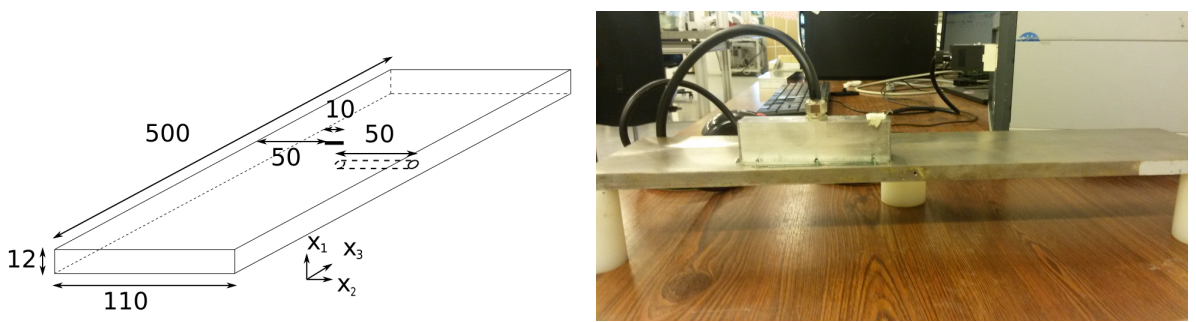

Figure 7: Left: scheme of the plate with defects. Right: experimental setup.

650

consequently the emission and reception matrices $E$ and $R$, are not invertible when two distinct propagating modes are associated with the same wave number. It is well-known that at high frequencies $\omega$ the two eigenvalues $\beta_{0}(\omega)$ and $\beta_{1}(\omega)$ (which correspond to the first and second Lamb modes $A_{0}$ and $S_{0}$ ) actu655 ally become very close, which deteriorates the quality of the identification. This is why, if $\left|\left(\beta_{0}-\beta_{1}\right) / \beta_{0}\right|<10^{-5}$, only the first of these two modes is taken into account in the inversion procedure.

Remark 11. Our specimen is far from being a true waveguide: its dimension in the $x_{3}$ direction is rather small, so that it can hardly be considered as a wavegwith respect to time for a given source (element number 128 of the transducer) and when the defect is the cylindrical hole. We can clearly distinguish 3 different waves: the wave emitted by the source (blue arrow), the wave scattered by the defect (green arrow) and the wave reflected by the edge of the plate (red smallness of the plate length. Furthermore, the dimensions of the three zones of the specimen in the $x_{2}$ direction are rather small too, especially the cylindrical notch, so that it seems to contradict the $2 D$ assumption. However, eventhough the assumptions of a $2 D$ waveguide are violated somehow, we will see that the 670 identification of defects is acceptable.

In the figure 9, we can see the identification results obtained by our inversion procedure described in paragraph 5.3 (back-scattering case) from experimental data when the defect is the cylindrical hole. The chosen polarization is $t_{3}$. The 


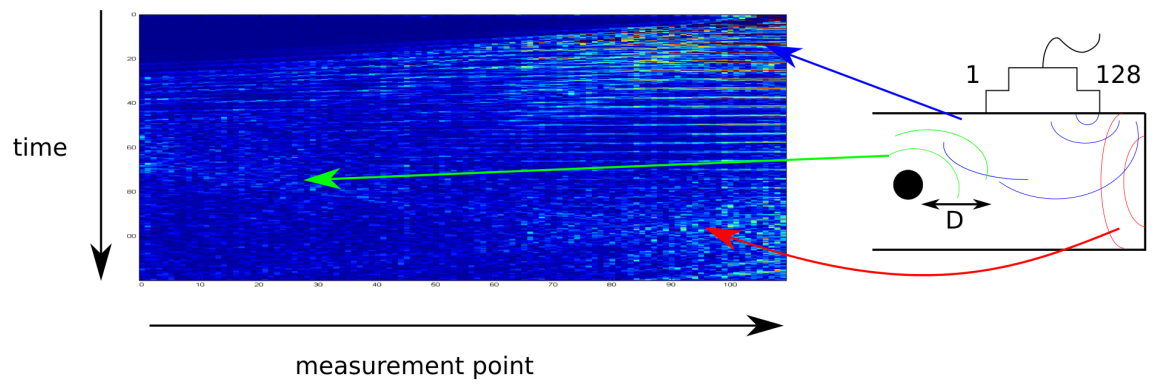

Figure 8: How the edge of the plate perturbs the measurements.

different pictures correspond to different distances $D$ between the transducer and the defect. More precisely, the element number 1 of the transducer is exactly located on the left edge of the picture and is fixed (the element number 128 is outside the picture), so that the defect is moving to the right of the picture when the distance $D$ increases. In the figure 10 , the same identification results are shown when the defect is the cylindrical notch, again with polarization $t_{3}$.

In all cases, the defect is identified. But we observe that the smaller is the
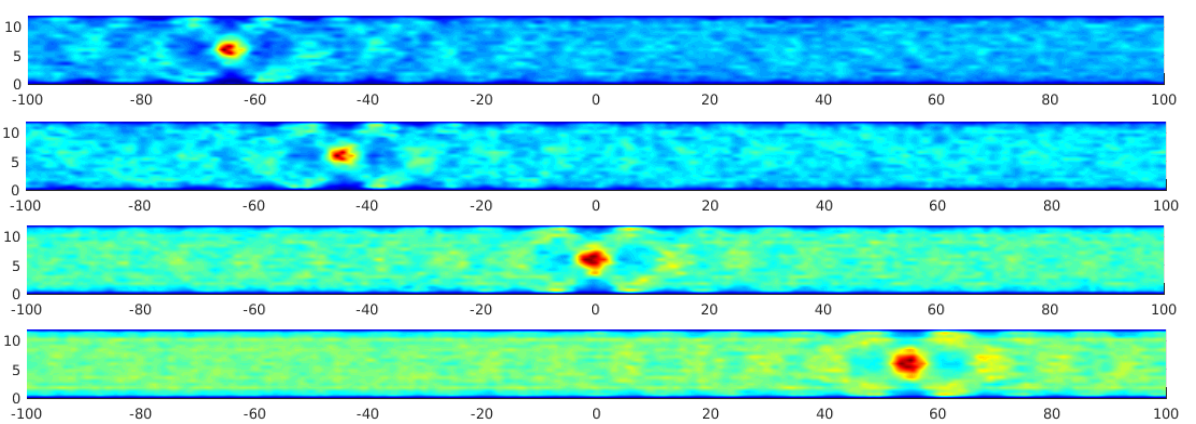

Figure 9: Identification of the cylindrical hole for $D=35 \mathrm{~mm}, D=55 \mathrm{~mm}, D=95 \mathrm{~mm}$ and $D=150 \mathrm{~mm}$.

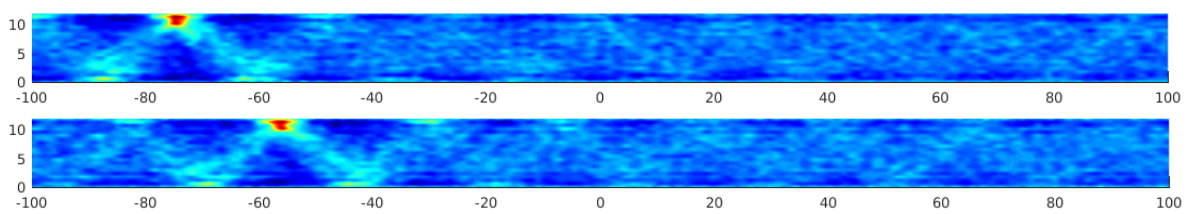

Figure 10: Identification of the cylindrical notch for $D=25 \mathrm{~mm}$ and $D=45 \mathrm{~mm}$.

distance between the transducer and the defect, the better is the resolution, which can be explained by some attenuation that is not taken into account 
in our model. In the last figure 11, we show an identification result for the cylindrical hole with the help of another transducer of lower mean frequency 1 $\mathrm{MHz}$, that is its bandwidth contains frequencies which correspond to 8 to 15 propagating modes. In addition, this transducer has 64 elements, the size of each element is $20 \mathrm{~mm} \times 0.9 \mathrm{~mm}$ in the $x_{2}$ and $x_{3}$ directions, respectively, the distance between two elements being $0.5 \mathrm{~mm}$, which implies that the distance between two sources/receivers in (4) is $\delta=1.4 \mathrm{~mm}$. We expect some degradation ${ }_{690}$ in the quality of the identification, because the support of the functions $f$ and $g$ is bigger and above all the frequencies are lower, which is what we observe for two different distances $D$.

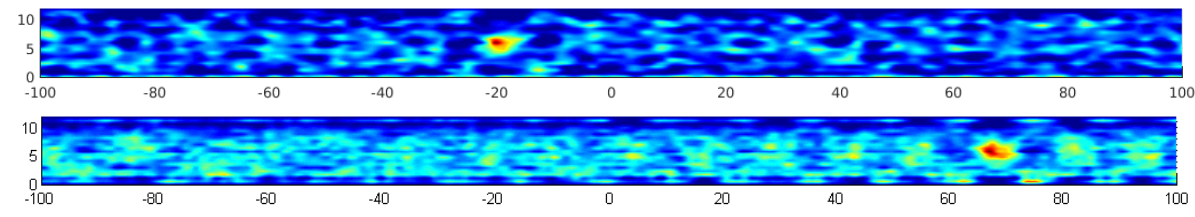

Figure 11: Identification of the cylindrical hole with a transducer of lower mean frequency for $D=80 \mathrm{~mm}$ and $D=170 \mathrm{~mm}$.

Remark 12. The data used in our numerical validations were noise free. The influence of noise has already been studied in [26] in the acoustic case for nu-

695 merical data and is actually the same in the elastic case. When experimental data are used, the presence of a small noise is inherent to the measurements, but we have seen that the reconstructions remain of good quality in that case.

\section{Conclusion}

To conclude, the numerical and experimental identification results that we obtained in the present study enable us to be confident in the feasibility of the Linear Sampling Method to retrieve a wide class of defects in the context of ultrasonic NDT of waveguides. For simplicity, all the theory in our paper was presented in 2D, but it is also valuable in 3D, as shown in [1, 2] as well as in [29]. However, the design of experiments with $3 \mathrm{D}$ waveguides, which are tubes rather than plates, raise some new questions related to the geometry and the location of the transducers. Those questions are partially addressed in [29. It would be certainly interesting to try the LSM for 3D geometries, both numerically and experimentally. More generally, from the experimental point of view, exploring other kinds of sensors and materials would be desirable in order to conclude on 710 the future of sampling methods in the context of NDT. 


\section{Appendix A: The fundamental solution for a Dirac on the boundary}

In this appendix, for $y=\left(d, y_{3}\right)$ we establish an expression of the solution $\mathcal{G}_{u}(\cdot, y)$ to the problem

$$
\left\{\begin{array}{clll}
\operatorname{div}\left(\sigma\left(\mathcal{G}_{u}(\cdot, y)\right)+\rho \omega^{2} \mathcal{G}_{u}(\cdot, y)\right. & =0 & & \text { in } \Omega_{R} \\
\sigma\left(\mathcal{G}_{u}(\cdot, y)\right) \boldsymbol{\nu} & =\delta_{y_{3}} \mathbf{e}_{1} & & \text { on } \Gamma_{d, R} \\
\sigma\left(\mathcal{G}_{u}(\cdot, y)\right) \boldsymbol{\nu} & = & & \text { on } \Gamma_{0, R} \\
T_{ \pm} \mathcal{G}_{Y}(\cdot, y) & = \pm \mathcal{G}_{X}(\cdot, y) & & \text { on } S_{ \pm R}
\end{array}\right.
$$

It is readily seen that the corresponding extended fundamental solution $\mathcal{G}(\cdot, y)$ 715 satisfies the evolution equation

$$
\left\{\begin{array}{ccc}
\frac{\partial}{\partial x_{3}} \mathcal{G}(\cdot, y)=\left(\begin{array}{cc}
0 & F_{Y} \\
F_{X} & 0
\end{array}\right) \mathcal{G}(\cdot, y) & \text { in } & W_{R} \\
\sigma_{11}\left(\mathcal{G}_{Y}(\cdot, y)\right)=\delta_{y_{3}}, \quad \mathrm{t}_{1}\left(\mathcal{G}_{Y}(\cdot, y)\right)=0 & \text { on } & \Gamma_{d, R} \\
\sigma_{11}\left(\mathcal{G}_{Y}(\cdot, y)\right)=0, \quad \mathrm{t}_{1}\left(\mathcal{G}_{Y}(\cdot, y)\right)=0 & \text { on } & \Gamma_{0, R} \\
T_{ \pm} \mathcal{G}_{Y}(\cdot, y)= \pm \mathcal{G}_{X}(\cdot, y) & \text { on } & S_{ \pm R}
\end{array}\right.
$$

We will need the following lemma.

Lemma 13. For sufficiently smooth $\mathcal{X}=\left(t_{1}, u_{3}\right)^{T}, \tilde{\mathcal{X}}=\left(\tilde{t}_{1}, \tilde{u}_{3}\right)^{T}, \mathcal{Y}=$ $\left(u_{1}, t_{3}\right)^{T}, \tilde{\mathcal{Y}}=\left(\tilde{u}_{1}, \tilde{t}_{3}\right)^{T}$, we have

$$
\begin{gathered}
\left(F_{Y} \mathcal{Y} \mid \tilde{\mathcal{Y}}\right)_{S}-\left(\mathcal{Y} \mid F_{Y} \tilde{\mathcal{Y}}\right)_{S} \\
=-\sigma_{11}(\mathcal{Y})(d) \tilde{u}_{1}(d)+\sigma_{11}(\mathcal{Y})(0) \tilde{u}_{1}(0)+\sigma_{11}(\tilde{\mathcal{Y}})(d) u_{1}(d)-\sigma_{11}(\tilde{\mathcal{Y}})(0) u_{1}(0)
\end{gathered}
$$

720 and

$$
\left(F_{X} \mathcal{X} \mid \tilde{\mathcal{X}}\right)_{S}-\left(\mathcal{X} \mid F_{X} \tilde{\mathcal{X}}\right)_{S}=t_{1}(d) \tilde{u}_{3}(d)-t_{1}(0) \tilde{u}_{3}(0)-\tilde{t}_{1}(d) u_{3}(d)+\tilde{t}_{1}(0) u_{3}(0) .
$$

Proof. From the definition of $F_{Y}$ given by (7), we have

$$
\left(F_{Y} \mathcal{Y} \mid \tilde{\mathcal{Y}}\right)_{S}=-\int_{0}^{d}\left(\partial_{x_{1}} \sigma_{11}(\mathcal{Y})+\rho \omega^{2} u_{1}\right) \tilde{u}_{1} d s-\int_{0}^{d}\left(\alpha \partial_{x_{1}} u_{1}+\frac{\alpha}{\lambda} t_{3}\right) \tilde{t}_{3} d s .
$$

By using the integration by parts formula, we have

$$
\int_{0}^{d} \partial_{x_{1}} \sigma_{11}(\mathcal{Y}) \tilde{u}_{1} d s=-\int_{0}^{d} \sigma_{11}(\mathcal{Y}) \partial_{x_{1}} \tilde{u}_{1} d s+\sigma_{11}(\mathcal{Y})(d) \tilde{u}_{1}(d)-\sigma_{11}(\mathcal{Y})(0) \tilde{u}_{1}(0) .
$$

In addition, in view of $(8), \alpha \tilde{t}_{3}=2 \mu(1+\alpha) \partial_{x_{1}} \tilde{u}_{1}-\sigma_{11}(\tilde{\mathcal{Y}})$, we hence obtain

$$
\begin{gathered}
\left(F_{Y} \mathcal{Y} \mid \tilde{\mathcal{Y}}\right)_{S}=-\sigma_{11}(\mathcal{Y})(d) \tilde{u}_{1}(d)+\sigma_{11}(\mathcal{Y})(0) \tilde{u}_{1}(0) \\
+\int_{0}^{d}\left(\sigma_{11}(\mathcal{Y}) \partial_{x_{1}} \tilde{u}_{1}+\sigma_{11}(\tilde{\mathcal{Y}}) \partial_{x_{1}} u_{1}-2 \mu(1+\alpha) \partial_{x_{1}} u_{1} \partial_{x_{1}} \tilde{u}_{1}-\frac{\alpha}{\lambda} t_{3} \tilde{t}_{3}-\rho \omega^{2} u_{1} \tilde{u}_{1}\right) d s
\end{gathered}
$$


725 We notice that the integral term in the right-hand side is symmetric with respect to $\mathcal{Y}$ and $\tilde{\mathcal{Y}}$, so that we obtain the first equality. From the definition of $F_{X}$ (see again (7), we have

$$
\left(F_{X} \mathcal{X} \mid \tilde{\mathcal{X}}\right)_{S}=\int_{0}^{d}\left(\frac{t_{1}}{\mu}-\partial_{x_{1}} u_{3}\right) \tilde{t}_{1} d s+\int_{0}^{d}\left(\partial_{x_{1}} t_{1}+\rho \omega^{2} u_{3}\right) \tilde{u}_{3} d s
$$

which by using the integration by parts formula leads to

$$
\begin{gathered}
\left(F_{X} \mathcal{X} \mid \tilde{\mathcal{X}}\right)_{S}=t_{1}(d) \tilde{u}_{3}(d)-t_{1}(0) \tilde{u}_{3}(0) \\
+\int_{0}^{d}\left(-\left(\partial_{x_{1}} u_{3} \tilde{t}_{1}+\partial_{x_{1}} \tilde{u}_{3} t_{1}\right)+\frac{1}{\mu} t_{1} \tilde{t}_{1}+\rho \omega^{2} u_{3} \tilde{u}_{3}\right) d s .
\end{gathered}
$$

730

Again the second equality stems from the symmetry of the integral term of the right-hand side with respect to $\mathcal{X}$ and $\tilde{\mathcal{X}}$.

In what follows, $\mathcal{D}(\mathbb{R})$ will denote the space of compactly supported and infinitely smooth functions on $\mathbb{R}$, while $\mathcal{D}^{\prime}(\mathbb{R})$ will denote the space of distributions on $\mathbb{R}$. By multiplying the two equations of $(55)$ by $\tilde{\mathbf{X}}_{n}\left(x_{1}, x_{3}\right)=\psi\left(x_{3}\right) \boldsymbol{\mathcal { X }}_{n}\left(x_{1}\right)$ and $\tilde{\mathbf{Y}}_{n}\left(x_{1}, x_{3}\right)=\psi\left(x_{3}\right) \mathcal{Y}_{n}\left(x_{1}\right)$, where $\psi \in \mathcal{D}(\mathbb{R})$ with $\operatorname{supp}(\psi) \subset(-R, R)$ and by integrating in $W$, we obtain

$$
\left\{\begin{array}{l}
\int_{W} \frac{\partial \mathcal{G}_{X}}{\partial x_{3}} \tilde{\mathbf{Y}}_{n} d x=\int_{W}\left(F_{Y} \mathcal{G}_{Y}\right) \tilde{\mathbf{Y}}_{n} d x \\
\int_{W} \frac{\partial \mathcal{G}_{Y}}{\partial x_{3}} \tilde{\mathbf{X}}_{n} d x=\int_{W}\left(F_{X} \mathcal{G}_{X}\right) \tilde{\mathbf{X}}_{n} d x
\end{array}\right.
$$

that is

$$
\left\{\begin{array}{c}
\int_{W} \mathcal{G}_{X} \frac{\partial \tilde{\mathbf{Y}}_{n}}{\partial x_{3}} d x+\int_{W}\left(F_{Y} \mathcal{G}_{Y}\right) \tilde{\mathbf{Y}}_{n} d x=0 \\
\int_{W} \mathcal{G}_{Y} \frac{\partial \tilde{\mathbf{X}}_{n}}{\partial x_{3}} d x+\int_{W}\left(F_{X} \mathcal{G}_{X}\right) \tilde{\mathbf{X}}_{n} d x=0,
\end{array}\right.
$$

By using the first equality of Lemma 13 , we have

740

$$
\begin{aligned}
& \int_{W}\left(F_{Y} \mathcal{G}_{Y}\right) \tilde{\mathbf{Y}}_{n} d x=\int_{\mathbb{R}}\left(F_{Y} \mathcal{G}_{Y} \mid \tilde{\mathbf{Y}}_{n}\right)_{S_{x_{3}}} d x_{3}=\int_{\mathbb{R}}\left(\mathcal{G}_{Y} \mid F_{Y} \mathcal{Y}_{n}\right)_{S_{x_{3}}} \psi\left(x_{3}\right) d x_{3} \\
& -\int_{\Gamma_{d}} \sigma_{11}\left(\mathcal{G}_{Y}\right)\left(d, x_{3}\right) u_{1}^{n}(d) \psi\left(x_{3}\right) d s+\int_{\Gamma_{0}} \sigma_{11}\left(\mathcal{G}_{Y}\right)\left(0, x_{3}\right) u_{1}^{n}(0) \psi\left(x_{3}\right) d s \\
& +\int_{\Gamma_{d}} \sigma_{11}\left(\mathcal{Y}_{n}\right)(d) \mathcal{G}_{u_{1}}\left(d, x_{3}\right) \psi\left(x_{3}\right) d s-\int_{\Gamma_{0}} \sigma_{11}\left(\mathcal{Y}_{n}\right)(0) \mathcal{G}_{u_{1}}\left(0, x_{3}\right) \psi\left(x_{3}\right) d s
\end{aligned}
$$

The system (9) satisfied by the guided modes implies that $F_{Y} \mathcal{Y}_{n}=i \beta_{n} \mathcal{X}_{n}$ and $\sigma_{11}\left(\mathcal{Y}_{n}\right)(d)=\sigma_{11}\left(\mathcal{Y}_{n}\right)(0)=0$. In addition, from the boundary conditions satisfied by $\sigma_{11}\left(\mathcal{G}_{Y}\right)$ on $\Gamma_{d}$ and $\Gamma_{0}$ in $(55)$, we end up with

$$
\int_{W}\left(F_{Y} \mathcal{G}_{Y}\right) \tilde{\mathbf{Y}}_{n} d x=i \beta_{n} \int_{\mathbb{R}}\left(\mathcal{G}_{Y} \mid \mathcal{X}_{n}\right)_{S_{x_{3}}} \psi\left(x_{3}\right) d x_{3}-u_{1}^{n}(d) \psi\left(y_{3}\right) .
$$


By using the second inequality of Lemma 13 , we have

$$
\begin{aligned}
\int_{W}\left(F_{X} \mathcal{G}_{X}\right) \tilde{\mathbf{X}}_{n} d x=\int_{\mathbb{R}}\left(F_{X} \mathcal{G}_{X} \mid \tilde{\mathbf{X}}_{n}\right)_{S_{x_{3}}} d x_{3}=\int_{\mathbb{R}}\left(\mathcal{G}_{X} \mid F_{X} \mathcal{X}_{n}\right)_{S_{x_{3}}} \psi\left(x_{3}\right) d x_{3} \\
\quad+\int_{\Gamma_{d}} \mathcal{G}_{t_{1}}\left(d, x_{3}\right) u_{3}^{n}(d) \psi\left(x_{3}\right) d s-\int_{\Gamma_{0}} \mathcal{G}_{t_{1}}\left(0, x_{3}\right) u_{3}^{n}(0) \psi\left(x_{3}\right) d s \\
\quad-\int_{\Gamma_{d}} t_{1}^{n}(d) \mathcal{G}_{u_{3}}\left(d, x_{3}\right) \psi\left(x_{3}\right) d s+\int_{\Gamma_{0}} t_{1}^{n}(0) \mathcal{G}_{u_{3}}\left(0, x_{3}\right) \psi\left(x_{3}\right) d s
\end{aligned}
$$

The system (9) satisfied by the guided modes implies that $F_{X} \mathcal{X}_{n}=i \beta_{n} \mathcal{Y}_{n}$ and $t_{1}^{n}(d)=t_{1}^{n}(0)=0$. From the boundary conditions satisfied by $\mathcal{G}_{t_{1}}$ on $\Gamma_{d}$ and $\Gamma_{0}$ in (55), we end up with

$$
\int_{W}\left(F_{X} \mathcal{G}_{X}\right) \tilde{\mathbf{X}}_{n} d x=i \beta_{n} \int_{\mathbb{R}}\left(\mathcal{G}_{X} \mid \mathcal{Y}_{n}\right)_{S_{x_{3}}} \psi\left(x_{3}\right) d x_{3} .
$$

Gathering the equations (56), 57) and 58, we obtain

$$
\left\{\begin{array}{c}
\int_{\mathbb{R}}\left(\mathcal{G}_{X} \mid \mathcal{Y}_{n}\right)_{S_{x_{3}}} \psi^{\prime}\left(x_{3}\right) d x_{3}+i \beta_{n} \int_{\mathbb{R}}\left(\mathcal{G}_{Y} \mid \mathcal{X}_{n}\right)_{S_{x_{3}}} \psi\left(x_{3}\right) d x_{3}=u_{1}^{n}(d) \psi\left(y_{3}\right) \\
\int_{\mathbb{R}}\left(\mathcal{G}_{Y} \mid \mathcal{X}_{n}\right)_{S_{x_{3}}} \psi^{\prime}\left(x_{3}\right) d x_{3}+i \beta_{n} \int_{\mathbb{R}}\left(\mathcal{G}_{X} \mid \mathcal{Y}_{n}\right)_{S_{x_{3}}} \psi\left(x_{3}\right) d x_{3}=0 .
\end{array}\right.
$$

Plugging the decomposition

$$
\left\{\begin{array}{l}
\mathcal{G}_{X}\left(x_{1}, x_{3}\right)=\sum_{m \in \mathbb{N}} a_{m}\left(x_{3}\right) \mathcal{X}_{m}\left(x_{1}\right) \\
\mathcal{G}_{Y}\left(x_{1}, x_{3}\right)=\sum_{m \in \mathbb{N}} b_{m}\left(x_{3}\right) \mathcal{Y}_{m}\left(x_{1}\right)
\end{array}\right.
$$

in (59) and using the biothogonality condition $\left(\mathcal{X}_{n} \mid \mathcal{Y}_{m}\right)_{S}=\delta_{m n}$ for $m, n \in \mathbb{N}$, we obtain

$$
\left\{\begin{array}{c}
\int_{\mathbb{R}} a_{n}\left(x_{3}\right) \psi^{\prime}\left(x_{3}\right) d x_{3}+i \beta_{n} \int_{\mathbb{R}} b_{n}\left(x_{3}\right) \psi\left(x_{3}\right) d x_{3}=u_{1}^{n}(d) \psi\left(y_{3}\right) \\
\int_{\mathbb{R}} b_{n}\left(x_{3}\right) \psi^{\prime}\left(x_{3}\right) d x_{3}+i \beta_{n} \int_{\mathbb{R}} a_{n}\left(x_{3}\right) \psi\left(x_{3}\right) d x_{3}=0
\end{array}\right.
$$

which is equivalent, in the distributional sense in $\mathbb{R}$, to

$$
\left\{\begin{array}{c}
-a_{n}^{\prime}+i \beta_{n} b_{n}=u_{1}^{n}(d) \delta_{y_{3}} \\
-b_{n}^{\prime}+i \beta_{n} a_{n}=0
\end{array}\right.
$$

which in particular implies that

$$
b_{n}^{\prime \prime}+\beta_{n}^{2} b_{n}=-i \beta_{n} u_{1}^{n}(d) \delta_{y_{3}}
$$

The general solution to that equation in $\mathcal{D}^{\prime}(\mathbb{R})$ is

$$
b_{n}=-\frac{u_{1}^{n}(d)}{2} e^{i \beta_{n}\left|x_{3}-y_{3}\right|}+A_{n} e^{i \beta_{n} x_{3}}+B_{n} e^{-i \beta_{n} x_{3}} .
$$


Since the radiation condition implies that $A_{n}=B_{n}=0$, we obtain that for all $n \in \mathbb{N}$,

$$
a_{n}\left(x_{3}\right)=-s\left(x_{3}-y_{3}\right) \frac{u_{1}^{n}(d)}{2} e^{i \beta_{n}\left|x_{3}-y_{3}\right|}, \quad b_{n}\left(x_{3}\right)=-\frac{u_{1}^{n}(d)}{2} e^{i \beta_{n}\left|x_{3}-y_{3}\right|} .
$$

In conclusion, the expression of $\mathcal{G}(\cdot, y)$ for $y=\left(d, y_{3}\right)$ is

$$
\left\{\begin{array}{c}
\mathcal{G}_{X}(x, y)=-\sum_{n \in \mathbb{N}} s\left(x_{3}-y_{3}\right) \frac{u_{1}^{n}(d)}{2} \mathcal{X}_{n}\left(x_{1}\right) e^{i \beta_{n}\left|x_{3}-y_{3}\right|} \\
\mathcal{G}_{Y}(x, y)=-\sum_{n \in \mathbb{N}} \frac{u_{1}^{n}(d)}{2} \mathcal{Y}_{n}\left(x_{1}\right) e^{i \beta_{n}\left|x_{3}-y_{3}\right|}
\end{array}\right.
$$

760

We hence see that the vector function $\mathcal{G}(\cdot, y)$ for $y=\left(d, y_{3}\right)$ coincides with the first column $G^{t_{1}}(\cdot, y)$ of the fundamental solution $G(\cdot, y)$ given by 18 .

\section{Appendix B: The invertibility of the matrix of modes}

This appendix provides a proof of Theorem 9, which is a necessary and sufficient condition to have $u_{1}^{n}(d)=0$ for some $n=0, \ldots, N-1$. Let us recall that the behaviour of our $2 \mathrm{D}$ waveguide is governed by isotropic elasticity, the density is $\rho$ and the Lamé constants are $\lambda$ and $\mu$. If $\beta$ is associated with a propagating mode, then $-\beta$ is as well. Since $\beta \neq 0$, we can then assume without loss of generality that $\beta>0$. From [39] (see also [33]), such $\beta$ is a solution to the following dispersion relation:

$$
\begin{gathered}
\left(\alpha_{s}^{2}-\beta^{2}\right)^{2} \cos \left(\frac{\alpha_{p} d}{2}+\kappa\right) \sin \left(\frac{\alpha_{s} d}{2}+\kappa\right) \\
+4 \beta^{2} \alpha_{p} \alpha_{s} \sin \left(\frac{\alpha_{p} d}{2}+\kappa\right) \cos \left(\frac{\alpha_{s} d}{2}+\kappa\right)=0,
\end{gathered}
$$

770 with

$$
\alpha_{s}^{2}=k_{s}^{2}-\beta^{2}, \quad \alpha_{p}^{2}=k_{p}^{2}-\beta^{2}, \quad k_{s}=\omega \sqrt{\frac{\rho}{\mu}}, \quad k_{p}=\omega \sqrt{\frac{\rho}{\lambda+2 \mu}},
$$

where $\kappa=0$ or $\kappa=\pi / 2$. The two components of the corresponding mode are given by

$$
u_{1}\left(x_{1}\right)=-2 A_{p} \alpha_{p} \sin \left(\alpha_{p}\left(x_{1}-\frac{d}{2}\right)+\kappa\right)+2 A_{s} \beta \sin \left(\alpha_{s}\left(x_{1}-\frac{d}{2}\right)+\kappa\right)
$$

and

$$
u_{3}\left(x_{1}\right)=2 A_{p} i \beta \cos \left(\alpha_{p}\left(x_{1}-\frac{d}{2}\right)+\kappa\right)+2 A_{s} i \alpha_{s} \cos \left(\alpha_{s}\left(x_{1}-\frac{d}{2}\right)+\kappa\right)
$$


where the amplitudes $A_{p}$ and $A_{s}$ are obtained with the help of the free boundary

condition, that is

$$
\left\{\begin{array}{l}
t \cos \left(\frac{\alpha_{p} d}{2}+\kappa\right) A_{p}-2 \beta \alpha_{s} \cos \left(\frac{\alpha_{s} d}{2}+\kappa\right) A_{s}=0 \\
2 \beta \alpha_{p} \sin \left(\frac{\alpha_{p} d}{2}+\kappa\right) A_{p}+t \sin \left(\frac{\alpha_{s} d}{2}+\kappa\right) A_{s}=0
\end{array}\right.
$$

with $t=\alpha_{s}^{2}-\beta^{2}$. The mode is said to be symmetric (resp. antisymmetric) if the component $u_{3}$ is symmetric (resp. antisymmetric) with respect to axis $x_{1}=d / 2$. The first case corresponds to $\kappa=0$, while the second one corresponds to $\kappa=\pi / 2$.

That the system (64) has a non trivial solution provides the dispersion relation (60). Under this condition, we can in general (provided the two terms in the right-hand side of (65) be not zero) choose the following amplitudes

$$
\left\{\begin{array}{l}
A_{p}=2 \beta \alpha_{s} \cos \left(\frac{\alpha_{s} d}{2}+\kappa\right) \\
A_{s}=t \cos \left(\frac{\alpha_{p} d}{2}+\kappa\right)
\end{array}\right.
$$

Plugging these amplitudes in 62, we obtain

$$
\begin{aligned}
u_{1}(d) & =-4 \beta \alpha_{s} \alpha_{p} \sin \left(\frac{\alpha_{p} d}{2}+\kappa\right) \cos \left(\frac{\alpha_{s} d}{2}+\kappa\right) \\
& +2 \beta t \sin \left(\frac{\alpha_{s} d}{2}+\kappa\right) \cos \left(\frac{\alpha_{p} d}{2}+\kappa\right) .
\end{aligned}
$$

It follows from the dispersion relation 60 that

$$
u_{1}(d)=\beta \sin \left(\frac{\alpha_{s} d}{2}+\kappa\right) \cos \left(\frac{\alpha_{p} d}{2}+\kappa\right)\left(\frac{\left(\alpha_{s}^{2}-\beta^{2}\right)^{2}}{\beta^{2}}+2 t\right),
$$

785 and lastly

$$
u_{1}(d)=t \frac{k_{s}^{2}}{\beta} \sin \left(\frac{\alpha_{s} d}{2}+\kappa\right) \cos \left(\frac{\alpha_{p} d}{2}+\kappa\right) .
$$

Let us now study the different cases when $u_{1}(d)=0$. From [66), this could occur in the 2 following cases.

1. The first case is $t=0$. This implies that $\alpha_{s}^{2}-\beta^{2}=0$, that is

$$
\beta=\omega \sqrt{\frac{\rho}{2 \mu}} .
$$

Besides, from (60), the fact that $t=0$ leads to

$$
4 \beta^{2} \alpha_{p} \alpha_{s} \sin \left(\alpha_{p} \frac{d}{2}+\kappa\right) \cos \left(\alpha_{s} \frac{d}{2}+\kappa\right)=0
$$


which from the fact that $\alpha_{s} \neq 0$ implies that

$$
\alpha_{p} \sin \left(\alpha_{p} \frac{d}{2}+\kappa\right) \cos \left(\alpha_{s} \frac{d}{2}+\kappa\right)=0 .
$$

Again we have to study 3 cases.

(a) The first sub-case is $\alpha_{p}=0$. We have then $\beta=k_{p}=k_{s} / \sqrt{2}$, which in not possible since $\lambda>0$.

(b) The second sub-case is $\sin \left(\alpha_{p} d / 2+\kappa\right)=0$. Then there exists $p \in \mathbb{N}$ such that

$$
\beta^{2}=\omega^{2} \frac{\rho}{\lambda+2 \mu}-\frac{4(p \pi-\kappa)^{2}}{d^{2}} .
$$

This implies that $\beta=k_{s} / \sqrt{2} \leq k_{p}$, which is impossible since $\lambda>0$

(c) The third sub-case is $\cos \left(\alpha_{s} d / 2+\kappa\right)=0$. This case is also impossible. Indeed, if we substitute $t=0$ and $\cos \left(\alpha_{s} d / 2+\kappa\right)=0$ in the free boundary condition 64 , we can take $A_{p}=0$ and $A_{s}=1$. It follows from 62 that

$$
u_{1}(d)=2 \beta \sin \left(\frac{\alpha_{s} d}{2}+\kappa\right) \neq 0
$$

In conclusion, the case $t=0$ is impossible.

2. The other case is $\sin \left(\alpha_{s} d / 2+\kappa\right) \cos \left(\alpha_{p} d / 2+\kappa\right)=0$.

Then, the dispersion relation becomes

$$
4 \beta^{2} \alpha_{p} \alpha_{s} \sin \left(\frac{\alpha_{p} d}{2}+\kappa\right) \cos \left(\frac{\alpha_{s} d}{2}+\kappa\right)=0 .
$$

Since $\beta>0$, we have again 3 cases to consider:

(a) The first sub-case is $\sin \left(\alpha_{p} d / 2+\kappa\right) \cos \left(\alpha_{s} d / 2+\kappa\right)=0$. This identity, together with $\sin \left(\alpha_{s} d / 2+\kappa\right) \cos \left(\alpha_{p} d / 2+\kappa\right)=0$, implies that either

$$
\sin \left(\frac{\alpha_{s} d}{2}+\kappa\right)=\sin \left(\frac{\alpha_{p} d}{2}+\kappa\right)=0
$$

or

$$
\cos \left(\frac{\alpha_{s} d}{2}+\kappa\right)=\cos \left(\frac{\alpha_{p} d}{2}+\kappa\right)=0 .
$$

We obtain that there exists $(p, q) \in \mathbb{N}^{2}, p$ and $q$ being both odd or both even such that

$$
\left\{\begin{array}{l}
\beta^{2}=\omega^{2} \frac{\rho}{\mu}-\frac{(p \pi-2 \kappa)^{2}}{d^{2}} \\
\beta^{2}=\omega^{2} \frac{\rho}{\lambda+2 \mu}-\frac{(q \pi-2 \kappa)^{2}}{d^{2}} .
\end{array}\right.
$$


(b) The second sub-case is $\alpha_{p}=0$, that is

$$
\beta=\omega \sqrt{\frac{\rho}{\lambda+2 \mu}} .
$$

Besides, since $\sin \left(\alpha_{s} d / 2+\kappa\right) \cos \left(\alpha_{p} d / 2+\kappa\right)=0$, either $\kappa=\pi / 2$ or $\sin \left(\alpha_{s} d / 2+\kappa\right)=0$. From (62), (63) and (65), we note that if $\kappa=\pi / 2$, then $A_{s}=0$ and therefore the two components of the displacement vanish, which we exclude. We hence infer that $\sin \left(\alpha_{s} d / 2+\kappa\right)=0$ and $\kappa=0$. That $\sin \left(\alpha_{s} d / 2\right)=0$ implies that there exists $m \in \mathbb{N}$ such that

$$
\beta^{2}=\omega^{2} \frac{\rho}{\mu}-\left(\frac{2 m \pi}{d}\right)^{2} .
$$

We notice that such case is a particular case of (67), with $\kappa=0$, $p=2 m$ and $q=0$.

(c) The third sub-case is $\alpha_{s}=0$, that is

$$
\beta=\omega \sqrt{\frac{\rho}{\mu}} .
$$

Besides, since $\sin \left(\alpha_{s} d / 2+\kappa\right) \cos \left(\alpha_{p} d / 2+\kappa\right)=0$, either $\kappa=0$ or $\cos \left(\alpha_{p} d / 2+\kappa\right)=0$. From (62), 63) and 65), we note that if $\kappa=0$, then $A_{p}=0$ and therefore the two components of the displacement vanish, which we exclude. We hence have $\cos \left(\alpha_{p} d / 2+\kappa\right)=0$ and $\kappa=\pi / 2$, which implies there exists $m \in \mathbb{N}$ such that

$$
\beta^{2}=\omega^{2} \frac{\rho}{\lambda+2 \mu}-\left(\frac{2 m \pi}{d}\right)^{2} .
$$

This never happens because $\beta=k_{s} \leq k_{p}$, which is impossible since $\lambda>0$

Conversely, the condition (67) implies that $T$ is not invertible. This completes the proof of Theorem 9

Remark 14. From the above analysis, it happens that when the condition (67) is satisfied, except when $\kappa=0, p$ is even and $q=0$, the corresponding $\beta_{n}$ is both associated with a symmetric and an antisymmetric mode.

\section{References}

[1] L. Bourgeois, F. Le Louer, E. Lunéville, On the use of Lamb modes in the linear sampling method for elastic waveguides, Inverse Problems 27 (5) (2011) 055001.

URL http://stacks . iop.org/0266-5611/27/i=5/a=055001

[2] L. Bourgeois, E. Lunéville, On the use of the linear sampling method to identify cracks in elastic waveguides, Inverse Problems 29 (2) (2013) 025017. URL http: //stacks . iop.org/0266-5611/29/i=2/a=025017 
840

[3] P. Huthwaite, Evaluation of inversion approaches for guided wave thickness mapping, Proceedings of the Royal Society of London A: Mathematical, Physical and Engineering Sciences 470. arXiv:http://rspa. royalsocietypublishing.org/content/470/2166/20140063.full.pdf, doi:10.1098/rspa.2014.0063.

845 URL http://rspa.royalsocietypublishing.org/content/470/2166/ 20140063

[4] A. Demma, P. Cawley, M. Lowe, A. Roosenbrand, B. Pavlakovic, The reflection of guided waves from notches in pipes: a guide for interpreting corrosion measurements, NDT \& E International 37 (3) (2004) 167 - 180.

850 doi:https://doi.org/10.1016/j.ndteint.2003.09.004

10 URL http://www.sciencedirect.com/science/article/pii/ S0963869503001269

[ [5] S. Fletcher, M. J. S. Lowe, M. Ratassepp, C. Brett, Detection of axial cracks in pipes using focused guided waves, Journal of Nondestructive Evaluation 31 (1) (2012) 56-64. doi:10.1007/s10921-011-0120-x. URL https://doi.org/10.1007/s10921-011-0120-x

口 [6] C. Willey, F. Simonetti, P. Nagy, G. Instanes, Guided wave tomography of pipes with high-order helical modes, NDT \& E International 65 (2014) 8 21. doi:https://doi.org/10.1016/j.ndteint.2014.03.010. URL http://www.sciencedirect.com/science/article/pii/ S0963869514000449

[7] J. Rao, M. Ratassepp, Z. Fan, Guided wave tomography based on full waveform inversion, IEEE Transactions on Ultrasonics, Ferroelectrics, and 865 Frequency Control 63 (5) (2016) 737-745. doi:10.1109/TUFFC.2016. 2536144 .

n [8] S. Rodriguez, M. Deschamps, M. Castaings, E. Ducasse, Guided wave topological imaging of isotropic plates, Ultrasonics 54 (7) (2014) 1880 1890. doi:https://doi.org/10.1016/j.ultras.2013.10.001.

1) URL http://www.sciencedirect.com/science/article/pii/ 870 S0041624X13002862

[9] C. Holmes, B. W. Drinkwater, P. D. Wilcox, Post-processing of the full matrix of ultrasonic transmit-receive array data for nondestructive evaluation, NDT \& E International 38 (8) (2005) $701-711$. doi:http://dx.doi.org/10.1016/j.ndteint.2005.04.002.

875 URL http://wWw.sciencedirect.com/science/article/pii/ S0963869505000721

[10] L. L. Jeune, S. Robert, E. L. Villaverde, C. Prada, Plane wave imaga ing for ultrasonic non-destructive testing: Generalization to multimodal imaging, Ultrasonics 64 (Supplement C) (2016) 128 - 138. doi:https://doi.org/10.1016/j.ultras.2015.08.008 

S0041624X15002188

[11] D. Colton, A. Kirsch, A simple method for solving inverse scattering problems in the resonance region, Inverse Problems 12 (1996) 383-393.

885

12] T. Arens, Linear sampling methods for $2 \mathrm{D}$ inverse elastic wave scattering, Inverse Problems 17 (5) (2001) 1445-1464. doi:10.1088/0266-5611/17/ $5 / 314$

URL http://dx.doi.org/10.1088/0266-5611/17/5/314

13] A. Charalambopoulos, D. Gintides, K. Kiriaki, The linear sampling method for non-absorbing penetrable elastic bodies, Inverse Problems 19 (3) (2003) 549-561. doi:10.1088/0266-5611/19/3/305 URL http://dx.doi.org/10.1088/0266-5611/19/3/305

[14] D. Colton, H. Haddar, P. Monk, The linear sampling method for solving the electromagnetic inverse scattering problem, SIAM J. Sci. Comput. 24 (3) (2002) 719-731. doi:10.1137/S1064827501390467. URL http://dx.doi.org/10.1137/S1064827501390467

[15] H. Haddar, P. Monk, The linear sampling method for solving the electromagnetic inverse medium problem, Inverse Problems 18 (3) (2002) 891-906. doi:10.1088/0266-5611/18/3/323. URL http://dx.doi.org/10.1088/0266-5611/18/3/323

[16] Y. Xu, C. Mawata, W. Lin, Generalized dual space indicator method for underwater imaging, Inverse Problems 16 (6) (2000) 1761-1776. URL http://stacks .iop.org/0266-5611/16/i=6/a=311

[17] A. Charalambopoulos, D. Gintides, K. Kiriaki, A. Kirsch, The factorization method for an acoustic wave guide, 7th Int. Workshop on Mathematical Methods in Scattering Theory and Biomedical Engineering (2006) 120-127.

[18] L. Bourgeois, E. Lunéville, The linear sampling method in a waveguide: a modal formulation, Inverse Problems 24 (1) (2008) 015018. doi:10.1088/ 0266-5611/24/1/015018.

910 URL http://dx.doi.org/10.1088/0266-5611/24/1/015018

[19] P. Monk, V. Selgas, Sampling type methods for an inverse waveguide problem, Inverse Problems and Imaging 6 (4) (2012) 709-747.

[20] L. Borcea, D.-L. Nguyen, Imaging with electromagnetic waves in termi-

nating waveguides, Inverse Probl. Imaging 10 (4) (2016) 915-941. doi:

915 10.3934/ipi.2016027

URL http://dx.doi.org/10.3934/ipi.2016027

[21] F. Cakoni, D. Colton, The linear sampling method for cracks, Inverse Problems 19 (2) (2003) 279-295. doi:10.1088/0266-5611/19/2/303.

URL http://dx.doi.org/10.1088/0266-5611/19/2/303 
[22] F. Ben Hassen, Y. Boukari, H. Haddar, Application of the linear sampling method to identify cracks with impedance boundary conditions, Inverse

I Probl. Sci. Eng. 21 (2) (2013) 210-234. doi:10.1080/17415977.2012. 686997

URL http://dx.doi.org/10.1080/17415977.2012.686997

[23] F. Pourahmadian, B. B. Guzina, H. Haddar, Generalized linear sampling method for elastic-wave sensing of heterogeneous fractures, Inverse Problems 33 (5) (2017) 055007, 32. doi:10.1088/1361-6420/33/5/055007. URL http://dx.doi.org/10.1088/1361-6420/33/5/055007

[24] Q. Chen, H. Haddar, A. Lechleiter, P. Monk, A sampling method for inverse scattering in the time domain, Inverse Problems 26 (8) (2010) 085001, 17. doi:10.1088/0266-5611/26/8/085001. URL http://dx.doi.org/10.1088/0266-5611/26/8/085001

[25] P. Monk, V. Selgas, An inverse acoustic waveguide problem in the time domain, Inverse Problems 32 (5) (2016) 055001. URL http://stacks .iop.org/0266-5611/32/i=5/a=055001

[26] V. Baronian, L. Bourgeois, A. Recoquillay, Imaging an acoustic waveguide

from surface data in the time domain, Wave Motion 66 (2016) 68-87. doi: $10.1016 / j$. wavemoti.2016.05.006 URL http://dx.doi.org/10.1016/j.wavemoti.2016.05.006

[27] L. Bourgeois, S. Fliss, On the identification of defects in a periodic waveguide from far field data Inverse Problems 30 (9) (2014) 095004, 31. URL https://doi.org/10.1088/0266-5611/30/9/095004

[28] H. Brezis, Functional analysis, Sobolev spaces and partial differential equations, Universitext, Springer, New York, 2011.

[29] A. Recoquillay, Méthodes d'échantillonnage appliquées à l'imagerie de défauts dans un guide d'ondes élastiques, Ph.D. thesis, Paris Saclay (2018). URL http://www. theses.fr/2018SACLY001

[30] V. Baronian, A. S. Bonnet-Ben Dhia, E. Lunéville, Transparent boundary conditions for the harmonic diffraction problem in an elastic waveguide, J. Comput. Appl. Math. 234 (6) (2010) 1945-1952. doi:10.1016/j.cam. 2009.08 .045

URL http://dx.doi.org/10.1016/j.cam.2009.08.045

[31] W. B. Fraser, Orthogonality relation for the Rayleigh-Lamb modes of vibration of a plate, J. Acoust. Soc. Am. 59 (1976) 215-216.

${ }_{955}[32]$ V. Pagneux, A. Maurel, Lamb wave propagation in elastic waveguides with variable thickness, Proc. R. Soc. Lond. Ser. A Math. Phys. Eng. Sci. 462 (2068) (2006) 1315-1339. doi:10.1098/rspa.2005.1612.

URL http://dx.doi.org/10.1098/rspa.2005.1612 
[33] V. Baronian, Couplage des méthodes modale et éléments finis pour la diffraction des ondes élastiques guidées, Ph.D. thesis, Ecole Polytechnique (2009).

[34] L. Bourgeois, E. Lunéville, The linear sampling method in a waveguide: a formulation based on modes, Journal of Physics: Conference Series 135 (2008) 012023.

[35] D. Colton, M. Piana, R. Potthast, A simple method using Morozov's discrepancy principle for solving inverse scattering problems, Inverse Problems 13 (1997) 1477-1493.

[36] A. Moitra, Super-resolution, extremal functions and the condition number of Vandermonde matrices, in: STOC'15-Proceedings of the 2015 ACM Symposium on Theory of Computing, ACM, New York, 2015, pp. 821-830.

[37] A. Imperiale, S. Chatillon, P. Calmon, N. Leymarie, S. Imperiale, E. Demaldent, UT Simulation of Embedded Parametric Defects Using a Hybrid Model Based Upon Spectral Finite Element and Domain Decomposition Methods, Proceedings of the 19th World Conference on NonDestructive Testing.

[38] K. Jezzine, Approche modale pour la simulation globale des contrôles nondestructifs par ondes élastiques guidées, Ph.D. thesis, Université Bordeaux 1 (2006).

[39] D. Royer, E. Dieulesaint, Ondes élastiques dans les solides, Interaction of Mechanics and Mathematics, Masson, Paris, 1996, tome 1, Propagation libre et guidée. 